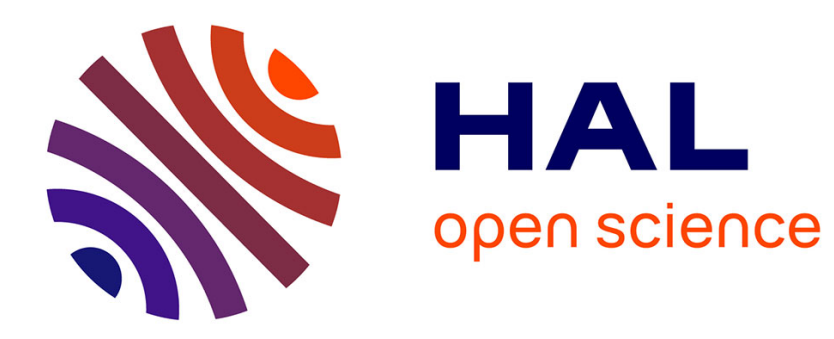

\title{
A Convergent Data Completion Algorithm Using Surface Integral Equations
}

Yosra Boukari, Houssem Haddar

\section{To cite this version:}

Yosra Boukari, Houssem Haddar. A Convergent Data Completion Algorithm Using Surface Integral Equations. Inverse Problems, 2015, pp.21. hal-01110005

\section{HAL Id: hal-01110005 \\ https://hal.inria.fr/hal-01110005}

Submitted on 27 Jan 2015

HAL is a multi-disciplinary open access archive for the deposit and dissemination of scientific research documents, whether they are published or not. The documents may come from teaching and research institutions in France or abroad, or from public or private research centers.
L'archive ouverte pluridisciplinaire HAL, est destinée au dépôt et à la diffusion de documents scientifiques de niveau recherche, publiés ou non, émanant des établissements d'enseignement et de recherche français ou étrangers, des laboratoires publics ou privés. 


\title{
A Convergent Data Completion Algorithm Using Surface Integral Equations
}

\author{
Yosra Boukari ${ }^{\mathrm{a}, \mathrm{b}}$ and Houssem Haddar ${ }^{\mathrm{b} *}$ \\ a ENIT-LAMSIN, Université de Tunis el-Manar, \\ BP 37, 1002, Tunis, Tunisia \\ b INRIA Saclay Ile de France/CMAP Ecole Polytechnique, \\ Route de Saclay, 91128, Palaiseau Cedex, France
}

December 9, 2014

\begin{abstract}
We propose and analyze a data completion algorithm based on the representation of the solution in terms of surface integral operators to solve the Cauchy problem for the Helmholtz or the Laplace equations. The proposed method is non iterative and intrinsically handle the case of noisy and incompatible data. In order to cope with the ill-posedness of the problem, our formulation is compatible with standard regularization methods associated with linear ill posed inverse problems and leads to convergent scheme. We numerically validate our method with different synthetic examples using a Tikhonov regularization.
\end{abstract}

\section{Introduction}

We are concerned with the design of robust and quick numerical algorithms that solve the ill-posed Cauchy problem in the framework of the Helmholtz or the Laplace equations. More specifically, we would like to determine the Cauchy data associated with a solution of the Helmholtz or the Laplace equation in a part of the boundary of a bounded domain from the knowledge of the Cauchy data on the complementary part. We consider the setting of a Lipschitz domain whose boundary can be split in two parts: an exterior boundary and an interior boundary that we denote by $\Gamma_{1}$ and $\Gamma_{2}$ respectively. The inverse problem under concern consists in reconstructing the interior Cauchy data on $\Gamma_{1}$ by knowing the Cauchy data on the exterior boundary $\Gamma_{2}$.

This problem is known to be severely ill-posed $[16,4]$ and only weak logarithmic conditional stability can be obtained in general (See for instance $[8,11,17,20,27,17]$ and references therein). On the numerical side, a large variety of methods has been proposed in the literature and it is hard to give an extensive overview of them. Let us however indicate some of them in order to put into perspective the method we shall introduce. A first class of method named quasi-reversibility relate missing data to the boundary values of a solution to a stable fourth order problem. It was originally proposed by Lattes and Lions in 1967 [24] and then extensively studied in [22, 7, 9, 14, 11]. This method is not iterative. However, the numerical implementation requires solvers for a fourth order problem, which may be computationally complex and expensive. The extension to Helmholtz type equations has not been studied yet.

In the class of iterative methods, several approaches have been proposed for the Laplace operator by either using a Kozlov [23] alternating scheme type [21, 6, 12, 5] or by minimizing an appropriate functional $[2,1,15]$. The case of Helmholtz equation has been addressed in only a few works $[3,25]$.

\footnotetext{
*Corresponding author. Email: haddar@cmap.polytechnique.fr
} 
In this paper, we propose a new method to solve this inverse problem. Using the boundary integral representation of the solution along with the trace of the solution and its normal derivative on $\Gamma_{1}$ and $\Gamma_{2}$, we derive a system of linear integral equation that is satisfied by the known and unknown Cauchy data. The solution of the inverse problem can be obtained by inverting the linear (matrix) operator that applies to the unknown Cauchy data. The main development here is the analysis of the structure of this operator. We observe for instance that it can be decomposed into an ill-posed component and a projection. This decomposition allows us to prove the injectivity of this operator and characterize the closure of its range. We then show that the system naturally handles the case of noisy and incompatible data, i.e. data that do not belong to the closure of the range of the operator to be inverted. Indeed, in our formulation the available Cauchy data are multiplied by an operator whose range closure is the same as for the operator to be inverted. This makes possible the use of classical regularization techniques for noisy data, such as the Tikhonov regularization. The main important consequence is a convergence result for the exact solution as the noise level goes to zero.

Some numerical results are then provided showing the efficiency of the method for different shapes of the domain, different values of the wave number and different noise levels. Note in addition that the implemented algorithm has the advantage to be fast as it does not rely on an iterative scheme.

Let us finally note that our approach shares similarities with the one proposed in [10] for the Laplace problem. However, for the latter, although also numerically efficient, one cannot ensure convergence towards the exact solution since it does not belong (in general) to the range of the used integral representation. For other conributions on solving the Cauchy problem using the integral representation and iterative methods, we refer the reader to $[25,18,19]$.

The outline of the article is as follows. In Section 2 we present the problem and derive the linear equation to be inverted. In Section 3 we analyze the obtained system and associated regularization. In Section 4 we provide numerical experiments that validate the proposed approach.

\section{A data completion algorithm based on integral equations}

We start this section by introducing the Cauchy problem associated with the Helmholtz equation (or the Laplace equation if the wave number is zero). Let $\Omega_{1} \subset \mathbf{R}^{m}$ be a bounded Lipschitz domain with connected complement and let $\Omega_{2} \subset \mathbf{R}^{m}$ be a Lipschitz subdomain of $\Omega_{1}$. We shall assume that $\Omega_{2}$ is strictly included in $\Omega_{1}$. We denote by $\Gamma_{1}$ and $\Gamma_{2}$ respectively the boundary of $\Omega_{1}$ and $\Omega_{2}$ and $\nu$ is the normal vector on $\Gamma_{1} \cup \Gamma_{2}$ directed to the exterior of $\Omega_{1}$ and $\Omega_{2}$ (see Figure $1)$. Let $u \in H^{1}\left(\Omega_{1} \backslash \Omega_{2}\right)$ solution of the following Helmholtz equation:

$$
\Delta u+k^{2} u=0 \quad \text { in } \Omega_{1} \backslash \Omega_{2},
$$

where $k$ denotes the wave number. Our data completion problem consists in determining $\left(u, \partial_{\nu} u\right)_{\mid \Gamma_{2}}$ while knowing $(f, g):=\left(u, \partial_{\nu} u\right)_{\mid \Gamma_{1}}$.

Our algorithm is based on the inversion of the surface representation of $u$ in terms of the boundary values $\left(u, \partial_{\nu} u\right)_{\mid \Gamma_{1}}$ and $\left(u, \partial_{\nu} u\right)_{\mid \Gamma_{2}}$. More precisely, since $u \in H^{1}\left(\Omega_{1} \backslash \Omega_{2}\right)$ satisfies the Helmholtz equation in $\Omega_{1} \backslash \Omega_{2}$, then $u$ has the following representation

$$
u(x)=-D L_{\Gamma_{1}}\left(u_{\mid \Gamma 1}\right)+S L_{\Gamma_{1}}\left(\partial_{\nu} u_{\mid \Gamma 1}\right)+D L_{\Gamma_{2}}\left(u_{\mid \Gamma 2}\right)-S L_{\Gamma_{2}}\left(\partial_{\nu} u_{\mid \Gamma 2}\right)
$$

for $x \in \Omega_{1} \backslash \overline{\Omega_{2}}$ where

$$
S L_{\Gamma_{i}} \psi(x):=\int_{\Gamma_{i}} \psi(y) \Phi(x, y) d s(y), \quad D L_{\Gamma_{i}} \varphi(x):=\int_{\Gamma_{i}} \varphi(y) \frac{\partial \Phi(x, y)}{\partial \nu(y)} d s(y),
$$




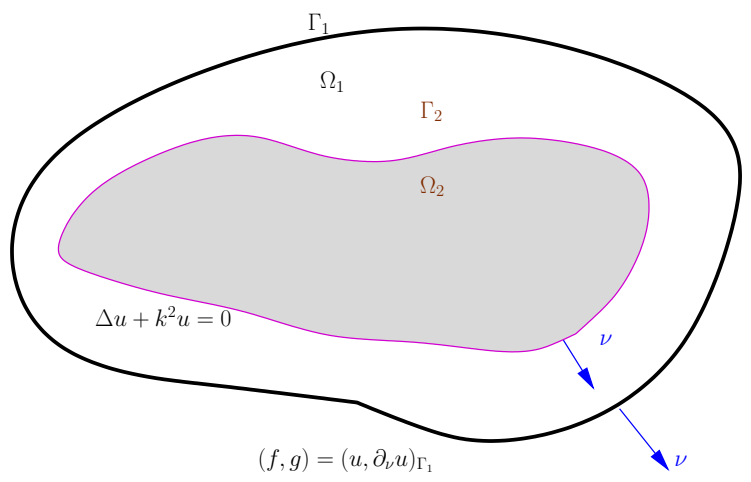

Figure 1: Representation of the domain

for $x \in \mathbf{R}^{m} \backslash \Gamma_{i}, i=1,2$, with $\Phi$ the Green function defined for $k \neq 0$ by

$$
\Phi(x, y):= \begin{cases}\frac{1}{4 \pi} \frac{e^{i k|x-y|}}{|x-y|} & m=3, \\ \frac{i}{4} H_{0}^{(1)}(k|x-y|) & m=2,\end{cases}
$$

for $x \neq y$ and $H_{0}^{(1)}$ being the Hankel function of the first kind of order zero and for $k=0, \Phi(x, y)$ is given by

$$
\Phi(x, y):= \begin{cases}\frac{1}{4 \pi} \frac{1}{|x-y|} & m=3, \\ \frac{-1}{2 \pi} \log (|x-y|) & m=2 .\end{cases}
$$

Considering the trace and the normal trace of $u$ on $\Gamma_{1}$ and using the trace properties of double and single layer potentials, we obtain the following identities

$$
\begin{aligned}
& f=-K_{\Gamma_{1}}(f)+\frac{f}{2}+S_{\Gamma_{1}}(g)+D L_{\Gamma_{2} \rightarrow \Gamma_{1}}\left(u_{\mid \Gamma_{2}}\right)-S L_{\Gamma_{2} \rightarrow \Gamma_{1}}\left(\partial_{\nu} u_{\mid \Gamma_{2}}\right), \\
& g=-T_{\Gamma_{1}}(f)+K_{\Gamma_{1}}^{\prime}(g)+\frac{g}{2}+T L_{\Gamma_{2} \rightarrow \Gamma_{1}}\left(u_{\mid \Gamma_{2}}\right)-D L_{\Gamma_{2} \rightarrow \Gamma_{1}}^{\prime}\left(\partial_{\nu} u_{\mid \Gamma_{2}}\right),
\end{aligned}
$$

where the boundary integral operators

$$
\begin{aligned}
S_{\Gamma_{i}}: H^{-1 / 2}\left(\Gamma_{i}\right) \rightarrow H^{1 / 2}\left(\Gamma_{i}\right), & K_{\Gamma_{i}}: H^{1 / 2}\left(\Gamma_{i}\right) \rightarrow H^{1 / 2}\left(\Gamma_{i}\right), \\
K_{\Gamma_{i}}^{\prime}: H^{-1 / 2}\left(\Gamma_{i}\right) \rightarrow H^{-1 / 2}\left(\Gamma_{i}\right), & T_{\Gamma_{i}}: H^{1 / 2}\left(\Gamma_{i}\right) \rightarrow H^{-1 / 2}\left(\Gamma_{i}\right),
\end{aligned}
$$

are defined for regular densities $\psi$ and $\varphi$ by

$$
\begin{array}{rrr}
S_{\Gamma_{i}} \psi(x):=\int_{\Gamma_{i}} \psi(y) \Phi(x, y) d s(y), & K_{\Gamma_{i}} \varphi(x):=\int_{\Gamma_{i}} \varphi(y) \frac{\partial \Phi(x, y)}{\partial \nu(y)} d s(y), \\
K_{\Gamma_{i}}^{\prime} \psi(x):=\int_{\Gamma_{i}} \psi(y) \frac{\partial \Phi(x, y)}{\partial \nu(x)} d s(y), & T_{\Gamma_{i}} \varphi(x):=\lim _{\epsilon \longrightarrow 0} \frac{\partial}{\partial \nu(x)} \int_{\Gamma_{i},|x-y|>\epsilon} \varphi(y) \frac{\partial \Phi(x, y)}{\partial \nu(y)} d s(y),
\end{array}
$$

for $x \in \Gamma_{i}$. The boundary integral operators for $i, j=1,2$ and $i \neq j$

$$
\begin{aligned}
S L_{\Gamma_{i} \rightarrow \Gamma_{j}}: H^{-1 / 2}\left(\Gamma_{i}\right) \longrightarrow H^{1 / 2}\left(\Gamma_{j}\right), & D L_{\Gamma_{i} \rightarrow \Gamma_{j}}: H^{1 / 2}\left(\Gamma_{i}\right) \longrightarrow H^{1 / 2}\left(\Gamma_{j}\right), \\
D L_{\Gamma_{i} \rightarrow \Gamma_{j}}^{\prime}: H^{-1 / 2}\left(\Gamma_{i}\right) \longrightarrow H^{-1 / 2}\left(\Gamma_{j}\right), & T L_{\Gamma_{i} \rightarrow \Gamma_{j}}: H^{1 / 2}\left(\Gamma_{i}\right) \longrightarrow H^{-1 / 2}\left(\Gamma_{j}\right),
\end{aligned}
$$

are defined for regular densities $\psi$ and $\varphi$ by

$$
\begin{gathered}
S L_{\Gamma_{i} \rightarrow \Gamma_{j}} \psi(x):=\int_{\Gamma_{i}} \psi(y) \Phi(x, y) d s(y), \quad D L_{\Gamma_{i} \rightarrow \Gamma_{j}} \varphi(x):=\int_{\Gamma_{i}} \varphi(y) \frac{\partial \Phi(x, y)}{\partial \nu(y)} d s(y), \\
D L_{\Gamma_{i} \rightarrow \Gamma_{j}}^{\prime} \psi(x):=\int_{\Gamma_{i}} \psi(y) \frac{\partial \Phi(x, y)}{\partial \nu(x)} d s(y), \quad T L_{\Gamma_{i} \rightarrow \Gamma_{j}} \varphi(x):=\frac{\partial}{\partial \nu(x)} \int_{\Gamma_{i}} \varphi(y) \frac{\partial \Phi(x, y)}{\partial \nu(y)} d s(y)
\end{gathered}
$$


for $x \in \Gamma_{j}$. We observe that, since $\Gamma_{i} \neq \Gamma_{j}$, the kernels of $D L_{\Gamma_{i} \rightarrow \Gamma_{j}}, S L_{\Gamma_{i} \rightarrow \Gamma_{j}}, D L_{\Gamma_{i} \rightarrow \Gamma_{j}}^{\prime}, T L_{\Gamma_{i} \rightarrow \Gamma_{j}}$ are smooth and therefore these operators are compact. Equations (5) and (6) can be written in a matrix form as

$$
-\left[\begin{array}{cc}
D L_{\Gamma_{2} \rightarrow \Gamma_{1}} & -S L_{\Gamma_{2} \rightarrow \Gamma_{1}} \\
T L_{\Gamma_{2} \rightarrow \Gamma_{1}} & -D L_{\Gamma_{2} \rightarrow \Gamma_{1}}^{\prime}
\end{array}\right]\left[\begin{array}{c}
u_{\mid \Gamma_{2}} \\
\partial_{\nu} u_{\mid \Gamma_{2}}
\end{array}\right]=\left[\begin{array}{cc}
-K_{\Gamma_{1}} & S_{\Gamma_{1}} \\
-T_{\Gamma_{1}} & K_{\Gamma_{1}}^{\prime}
\end{array}\right]\left[\begin{array}{c}
f \\
g
\end{array}\right]-\frac{1}{2}\left[\begin{array}{c}
f \\
g
\end{array}\right] .
$$

Using the Calderòn projector $P_{\Gamma_{1}}: H^{1 / 2}\left(\Gamma_{1}\right) \times H^{-1 / 2}\left(\Gamma_{1}\right) \longrightarrow H^{1 / 2}\left(\Gamma_{1}\right) \times H^{-1 / 2}\left(\Gamma_{1}\right)$ defined by

$$
P_{\Gamma_{1}}\left[\begin{array}{l}
f \\
g
\end{array}\right]:=\left[\begin{array}{cc}
-K_{\Gamma_{1}} & S_{\Gamma_{1}} \\
-T_{\Gamma_{1}} & K_{\Gamma_{1}}^{\prime}
\end{array}\right]\left[\begin{array}{l}
f \\
g
\end{array}\right]+\frac{1}{2}\left[\begin{array}{l}
f \\
g
\end{array}\right]
$$

(we recall that $P_{\Gamma_{1}}^{2}=P_{\Gamma_{1}},[26]$ ) and the operator $T_{\Gamma_{2} \rightarrow \Gamma_{1}}: H^{1 / 2}\left(\Gamma_{2}\right) \times H^{-1 / 2}\left(\Gamma_{2}\right) \longrightarrow H^{1 / 2}\left(\Gamma_{1}\right) \times$ $H^{-1 / 2}\left(\Gamma_{1}\right)$ defined by

$$
T_{\Gamma_{2} \rightarrow \Gamma_{1}}\left[\begin{array}{c}
\varphi \\
\psi
\end{array}\right]:=-\left[\begin{array}{ll}
D L_{\Gamma_{2} \rightarrow \Gamma_{1}} & -S L_{\Gamma_{2} \rightarrow \Gamma_{1}} \\
T L_{\Gamma_{2} \rightarrow \Gamma_{1}} & -D L_{\Gamma_{2} \rightarrow \Gamma_{1}}^{\prime}
\end{array}\right]\left[\begin{array}{l}
\varphi \\
\psi
\end{array}\right],
$$

we obtain the following first equation

$$
T_{\Gamma_{2} \rightarrow \Gamma_{1}}\left[\begin{array}{c}
u_{\mid \Gamma_{2}} \\
\partial_{\nu} u_{\mid \Gamma_{2}}
\end{array}\right]=\left(P_{\Gamma_{1}}-I_{\Gamma_{1}}\right)\left[\begin{array}{c}
f \\
g
\end{array}\right]
$$

where $I_{\Gamma_{i}}$ is the identity operator on $H^{1 / 2}\left(\Gamma_{i}\right) \times H^{-1 / 2}\left(\Gamma_{i}\right), i=1,2$. Similarly, considering the trace and the normal trace of $u$ on $\Gamma_{2}$, we obtain a second relationship between $(f, g)$ and $\left(u, \partial_{\nu} u\right)_{\Gamma_{2}}$, namely

$$
\begin{gathered}
u_{\mid \Gamma_{2}}=-D L_{\Gamma_{1} \rightarrow \Gamma_{2}}(f)+S L_{\Gamma_{1} \rightarrow \Gamma_{2}}(g)+K_{\Gamma_{2}}\left(u_{\mid \Gamma_{2}}\right)+\frac{u_{\mid \Gamma_{2}}}{2}-S_{\Gamma_{2}}\left(\partial_{\nu} u_{\mid \Gamma_{2}}\right) \\
\partial_{\nu} u_{\mid \Gamma_{2}}=-T L_{\Gamma_{1} \rightarrow \Gamma_{2}}(f)+D L_{\Gamma_{1} \rightarrow \Gamma_{2}}^{\prime}(g)+T_{\Gamma_{2}}\left(u_{\mid \Gamma_{2}}\right)-K_{\Gamma_{2}}^{\prime}\left(\partial_{\nu} u_{\mid \Gamma_{2}}\right)+\frac{\partial_{\nu} u_{\mid \Gamma_{2}}}{2}
\end{gathered}
$$

Therefore, we also have

$$
P_{\Gamma_{2}}\left[\begin{array}{c}
u_{\mid \Gamma_{2}} \\
\partial_{\nu} u_{\mid \Gamma_{2}}
\end{array}\right]=T_{\Gamma_{2} \rightarrow \Gamma_{1}}\left[\begin{array}{c}
f \\
g
\end{array}\right] .
$$

with $P_{\Gamma_{2}}$ and $T_{\Gamma_{1} \rightarrow \Gamma_{2}}$ defined similarly to $P_{\Gamma_{1}}$ and $T_{\Gamma_{2} \rightarrow \Gamma_{1}}$. By grouping (8) and (9) we end up with the following system

$$
\left[\begin{array}{c}
T_{\Gamma_{2} \rightarrow \Gamma_{1}} \\
P_{\Gamma_{2}}
\end{array}\right]\left[\begin{array}{c}
u_{\mid \Gamma_{2}} \\
\partial_{\nu} u_{\mid \Gamma_{2}}
\end{array}\right]=\left[\begin{array}{c}
\left(P_{\Gamma_{1}}-I_{\Gamma_{1}}\right) \\
T_{\Gamma_{1} \rightarrow \Gamma_{2}}
\end{array}\right]\left[\begin{array}{l}
f \\
g
\end{array}\right]
$$

In order to simplify the notation, we introduce $A: H^{1 / 2}\left(\Gamma_{2}\right) \times H^{-1 / 2}\left(\Gamma_{2}\right) \longrightarrow H^{1 / 2}\left(\Gamma_{1}\right) \times$ $H^{-1 / 2}\left(\Gamma_{1}\right) \times H^{1 / 2}\left(\Gamma_{2}\right) \times H^{-1 / 2}\left(\Gamma_{2}\right)$ defined as

$$
A\left[\begin{array}{c}
\varphi \\
\psi
\end{array}\right]=\left[\begin{array}{c}
T_{\Gamma_{2} \rightarrow \Gamma_{1}} \\
P_{\Gamma_{2}}
\end{array}\right]\left[\begin{array}{l}
\varphi \\
\psi
\end{array}\right]
$$

and $B: H^{1 / 2}\left(\Gamma_{1}\right) \times H^{-1 / 2}\left(\Gamma_{1}\right) \longrightarrow H^{1 / 2}\left(\Gamma_{1}\right) \times H^{-1 / 2}\left(\Gamma_{1}\right) \times H^{1 / 2}\left(\Gamma_{2}\right) \times H^{-1 / 2}\left(\Gamma_{2}\right)$ defined as

$$
B\left[\begin{array}{l}
\varphi \\
\psi
\end{array}\right]=\left[\begin{array}{c}
\left(P_{\Gamma_{1}}-I_{\Gamma_{1}}\right) \\
T_{\Gamma_{1} \rightarrow \Gamma_{2}}
\end{array}\right]\left[\begin{array}{l}
\varphi \\
\psi
\end{array}\right] .
$$


Then our inverse problem can be simply written as seeking for $(\varphi, \psi) \in H^{1 / 2}\left(\Gamma_{2}\right) \times H^{-1 / 2}\left(\Gamma_{2}\right)$ satisfying

$$
A\left[\begin{array}{l}
\varphi \\
\psi
\end{array}\right]=B\left[\begin{array}{l}
f \\
g
\end{array}\right] .
$$

The ill-posedness of this problem can be seen in the compactness of the operator $T_{\Gamma_{2} \rightarrow \Gamma_{1}}$ (all of its components are integral operators with smooth kernels). In order to apply a regularization strategy we should first investigate the injectivity of $A$ and its adjoint $A^{*}$. As we shall see, this property fails for the adjoint but is replaced with $\overline{\operatorname{Range}(A)}=\overline{\operatorname{Range}(B)}$. The latter also ensures that the right hand side of (13) is always in the closure of the range of $A$ for noisy data.

Remark 1. Let us notice that conceptually our method can be extended to the case where $\Gamma_{1}$ and $\Gamma_{2}$ are parts of the same boundary. However, the functional space setting has to modified to ensure proper continuity properties at the surface boundaries (see for instance [26] for trace spaces on open surfaces).

Remark 2. The method (as any boundary integral method) is not suited for inhomogeneous media. However, it can handle the case of piecewise constant backgrounds with known interfaces by incorporating the interfaces as parts of $\Gamma_{2}$. Another alternative would be to (numerically) compute the Green function of the background, but this would be very costly.

\section{Analysis of operators A and B}

We first need to characterize the range and the kernel of $T_{\Gamma_{i} \rightarrow \Gamma_{j}}, i \neq j$. To simplify the notation we introduce the following spaces $(i=1,2)$ :

$$
\begin{aligned}
& \mathcal{H}\left(\Omega_{i}\right):=\left\{u \in H^{1}\left(\Omega_{i}\right), \quad \Delta u+k^{2} u=0 \quad \text { in } \quad \Omega_{i}\right\} \\
& \tilde{\mathcal{H}}\left(\Omega_{i}\right):=\left\{u \in H_{l o c}^{1}\left(\mathbf{R}^{m} \backslash \Omega_{1}\right), \quad \Delta u+k^{2} u=0 \quad \text { in } \quad \mathbf{R}^{m} \backslash \Omega_{i} \quad \text { and } u \text { satisfies the } R . C .\right\}
\end{aligned}
$$

where R.C. refers to the Sommerfeld radiation condition which is defined for $k \neq 0$ as follows

$$
\lim _{r=|x| \rightarrow+\infty} r^{\frac{m-1}{2}}\left(\partial_{r} u-i k u\right)=0,
$$

uniformly in all directions $\hat{x}=\frac{x}{|x|}$. In the Laplace case, i.e. $k=0$, the R.C. simply refers here to $u(x)$ vanishing at $|x|=\infty$. The following classical notation will be used

$$
\begin{gathered}
v_{\mid \Gamma_{i}}^{ \pm}(x):=\lim _{h \rightarrow 0^{+}} v(x \pm h \nu) \quad \text { and } \partial_{\nu} v_{\mid \Gamma_{i}}^{ \pm}(x):=\lim _{h \rightarrow 0^{+}} \nu \cdot \nabla v(x \pm h \nu) \text { for } x \in \Gamma_{i} \\
{[v]_{\mid \Gamma_{i}}:=v_{\mid \Gamma_{i}}^{+}-v_{\mid \Gamma_{i}}^{-} \text {and }\left[\partial_{\nu} v\right]_{\mid \Gamma_{i}}:=\partial_{\nu} v_{\mid \Gamma_{i}}^{+}-\partial_{\nu} v_{\mid \Gamma_{i}}^{-} .}
\end{gathered}
$$

Lemma 3. The following identities hold.

$$
\begin{aligned}
\text { a) } \operatorname{Ker}\left(T_{\Gamma_{2} \rightarrow \Gamma_{1}}\right) & =\left\{\left(u, \partial_{\nu} u\right)_{\mid \Gamma_{2}} ; u \in \mathcal{H}\left(\Omega_{2}\right)\right\}=\operatorname{Ker}\left(P_{\Gamma_{2}}-I_{\Gamma_{2}}\right) . \\
\text { b) } \operatorname{Ker}\left(T_{\Gamma_{1} \rightarrow \Gamma_{2}}\right) & =\left\{\left(u, \partial_{\nu} u\right)_{\mid \Gamma_{1}} ; u \in \tilde{\mathcal{H}}\left(\Omega_{1}\right)\right\}=\operatorname{Ker}\left(P_{\Gamma_{1}}\right) .
\end{aligned}
$$

Proof. We start by proving $a)$. Let $(\varphi, \psi) \in H^{1 / 2}\left(\Gamma_{2}\right) \times H^{-1 / 2}\left(\Gamma_{2}\right)$ such that $T_{\Gamma_{2} \rightarrow \Gamma_{1}}(\varphi, \psi)=0$. We denote by $v$ the solution of the Helmholtz (or Laplace) equation in $\mathbf{R}^{m} \backslash \Gamma_{2}$ given by:

$$
v=-D L_{\Gamma_{2}}(\varphi)+S L_{\Gamma_{2}}(\psi), \quad \text { in } \quad \mathbf{R}^{m} \backslash \Gamma_{2} .
$$

Knowing that $T_{\Gamma_{2} \rightarrow \Gamma_{1}}(\varphi, \psi)=0$, it is clear that $\left(v, \partial_{\nu} v\right)$ vanishes on $\Gamma_{1}$. Using the unique continuation principle for radiating solutions, we get that $v=0$ in $\mathbf{R}^{m} \backslash \Omega_{2}$. Consequently, we obtain

$$
\left(v^{+}, \partial_{\nu} v^{+}\right)_{\mid \Gamma_{2}}=(0,0) \quad \text { on } \Gamma_{2} .
$$


Since $[v]_{\mid \Gamma_{2}}=-\varphi$ and $\left[\partial_{\nu} v\right]_{\mid \Gamma_{2}}=-\psi$, we get $(\varphi, \psi)=\left(v^{-}, \partial_{\nu} v^{-}\right)_{\mid \Gamma_{2}}$. This proves that $\operatorname{Ker}\left(T_{\Gamma_{2} \rightarrow \Gamma_{1}}\right) \subset$ $\left\{\left(u, \partial_{\nu} u\right)_{\mid \Gamma_{2}} ; u \in \mathcal{H}\left(\Omega_{2}\right)\right\}$.

For the reverse inclusion, consider $(\varphi, \psi)=\left(u, \partial_{\nu} u\right)_{\mid \Gamma_{2}}$ where $u \in \mathcal{H}\left(\Omega_{2}\right)$. For $x \in \mathbf{R}^{m} \backslash \overline{\Omega_{2}}$ we have, using the Green formula in $\Omega_{2}$,

$$
\int_{\Gamma_{2}} \partial_{\nu} u(y) \Phi(x, y) d s(y)-\int_{\Gamma_{2}} u(y) \partial_{\nu} \Phi(x, y) d s(y)=0 .
$$

Therefore, the function $v$ be defined by (15) satisfies $v=0$ in $\mathbf{R}^{m} \backslash \overline{\Omega_{2}}$. Hence

$$
\left(v, \partial_{\nu} v\right)_{\mid \Gamma_{1}}=0 \quad \text { and } \quad T_{\Gamma_{2} \rightarrow \Gamma_{1}}(\varphi, \psi)=(0,0) .
$$

Consequently $\operatorname{Ker}\left(T_{\Gamma_{2} \rightarrow \Gamma_{1}}\right)=\left\{\left(u, \partial_{\nu} u\right)_{\mid \Gamma_{2}} ; u \in \mathcal{H}\left(\Omega_{2}\right)\right\}$.

We now prove the second equality in a), i.e. $\operatorname{Ker}\left(T_{\Gamma_{2} \rightarrow \Gamma_{1}}\right)=\operatorname{Ker}\left(P_{\Gamma_{2}}-I_{\Gamma_{2}}\right)$. In fact we proved in the first part that if $(\varphi, \psi) \in \operatorname{Ker}\left(T_{\Gamma_{2} \rightarrow \Gamma_{1}}\right)$ then $(\varphi, \psi)=\left(v, \partial_{\nu} v\right)_{\mid \Gamma_{2}}$ where $v$ satisfies the Helmholtz equation inside $\Omega_{2}$ and can be represented as

$$
v=-D L_{\Gamma_{2}}\left(v_{\mid \Gamma_{2}}\right)+S L_{\Gamma_{2}}\left(\partial_{\nu} v_{\mid \Gamma_{2}}\right) \quad \text { in } \quad \Omega_{2} .
$$

Using the trace and the normal derivative trace of $v$ on $\Gamma_{2}$ we obtain

$$
\begin{gathered}
v_{\mid \Gamma_{2}}=-K_{\Gamma_{2}}\left(v_{\mid \Gamma_{2}}\right)+\frac{v_{\mid \Gamma_{2}}}{2}+S_{\Gamma_{2}}\left(\partial_{\nu} v_{\mid \Gamma_{2}}\right), \\
\partial_{\nu} v_{\mid \Gamma_{2}}=-T_{\Gamma_{2}}\left(v_{\mid \Gamma_{2}}\right)+K_{\Gamma_{2}}^{\prime}\left(\partial_{\nu} v_{\mid \Gamma_{2}}\right)+\frac{\partial_{\nu} v_{\mid \Gamma_{2}}}{2},
\end{gathered}
$$

which proves that $\left(v, \partial_{\nu} v\right)_{\mid \Gamma_{2}} \in \operatorname{Ker}\left(P_{\Gamma_{2}}-I_{\Gamma_{2}}\right)$. On the other hand let $(\varphi, \psi) \in \operatorname{Ker}\left(P_{\Gamma_{2}}-I_{\Gamma_{2}}\right)$ and let the function $v$ defined by (15).Using again trace formulas for double and single layer potentials, we obtain

$$
\left(\begin{array}{c}
v_{\mid \Gamma_{2}}^{+} \\
\partial_{\nu} v_{\mid \Gamma_{2}}^{+}
\end{array}\right)=\left(P_{\Gamma_{2}}-I_{\Gamma_{2}}\right)\left(\begin{array}{c}
\psi \\
\varphi
\end{array}\right)=\left(\begin{array}{l}
0 \\
0
\end{array}\right) .
$$

Therefore the function $v$ replaced by 0 in $\Omega_{2}$ is locally $H^{1}$ and is solution to the Helmholtz equation in $\mathbf{R}^{m}$. Unique continuation principle implies that $v$ is zero outside $\Omega_{2}$, which proves that $\left(v, \partial_{\nu} v\right)_{\mid \Gamma_{1}}$ is also zero. Consequently $(\varphi, \psi) \in \operatorname{Ker}\left(T_{\Gamma_{2} \rightarrow \Gamma_{1}}\right)$.

The proof of $b$ ) uses similar arguments as the proof of $a$ ) and is based on considering $v$ defined by (15) where $\Gamma_{2}$ is replaced with $\Gamma_{1}$, namely

$$
v=-D L_{\Gamma_{1}}(\varphi)+S L_{\Gamma_{1}}(\psi), \quad \text { in } \quad \mathbf{R}^{m} \backslash \Gamma_{1}
$$

for $(\varphi, \psi) \in H^{1 / 2}\left(\Gamma_{1}\right) \times H^{-1 / 2}\left(\Gamma_{1}\right)$. Assume that $T_{\Gamma_{1} \rightarrow \Gamma_{2}}(\varphi, \psi)=0$. Then $\left(v, \partial_{\nu} v\right)$ vanishes on $\Gamma_{2}$. Using the unique continuation principle for solutions of the Helmholtz equation inside $\Omega_{1}$ we get $v=0$ in $\Omega_{1}$. Consequently, we obtain

$$
\left(v^{-}, \partial_{\nu} v^{-}\right)_{\mid \Gamma_{1}}=(0,0) \quad \text { on } \Gamma_{1} .
$$

Since $[v]_{\mid \Gamma_{1}}=-\varphi$ and $\left[\partial_{\nu} v\right]_{\mid \Gamma_{1}}=-\psi$, we get $(\varphi, \psi)=-\left(v^{+}, \partial_{\nu} v^{+}\right)_{\mid \Gamma_{1}}$. This proves that $\operatorname{Ker}\left(T_{\Gamma_{1} \rightarrow \Gamma_{2}}\right) \subset\left\{\left(u, \partial_{\nu} u\right)_{\mid \Gamma_{1}} ; u \in \tilde{\mathcal{H}}\left(\Omega_{1}\right)\right\}$.

For the reverse inclusion, consider $(\varphi, \psi)=\left(u, \partial_{\nu} u\right)_{\mid \Gamma_{1}}$ where $u \in \tilde{\mathcal{H}}\left(\Omega_{1}\right)$. For $x \in \Omega_{1}$, using the fact that $u$ satisfies the radiation condition we get

$$
\int_{\Gamma_{1}} \partial_{\nu} u(y) \Phi(x, y) d s(y)-\int_{\Gamma_{1}} u(y) \partial_{\nu} \Phi(x, y) d s(y)=0 .
$$

Therefore, the function $v$ defined by (16) satisfies $v=0$ in $\Omega_{1}$. Hence

$$
\left(v, \partial_{\nu} v\right)_{\mid \Gamma_{2}}=0 \quad \text { and } \quad T_{\Gamma_{1} \rightarrow \Gamma_{2}}(\varphi, \psi)=(0,0) .
$$


Consequently $\operatorname{Ker}\left(T_{\Gamma_{1} \rightarrow \Gamma_{2}}\right)=\left\{\left(u, \partial_{\nu} u\right)_{\mid \Gamma_{1}} ; u \in \tilde{\mathcal{H}}\left(\Omega_{1}\right)\right\}$.

We now prove the second equality in b), i.e. $\operatorname{Ker}\left(T_{\Gamma_{1} \rightarrow \Gamma_{2}}\right)=\operatorname{Ker} P_{\Gamma_{1}}$. The first part of the proof of assertion b) shows that if $(\varphi, \psi) \in \operatorname{Ker}\left(T_{\Gamma_{2} \rightarrow \Gamma_{1}}\right)$ then $(\varphi, \psi)=-\left(v, \partial_{\nu} v\right)_{\mid \Gamma_{2}}$ where $v$ satisfies the Helmholtz equation outside $\Omega_{1}$ and can be represented as

$$
v=D L_{\Gamma_{2}}\left(v_{\mid \Gamma_{1}}\right)-S L_{\Gamma_{1}}\left(\partial_{\nu} v_{\mid \Gamma_{1}}\right) \quad \text { in } \quad \mathbf{R}^{m} \backslash \overline{\Omega_{1}} .
$$

Using the trace and the normal derivative trace of $v$ on $\Gamma_{1}$ we obtain

$$
\begin{aligned}
v_{\mid \Gamma_{1}} & =K_{\Gamma_{1}}\left(v_{\mid \Gamma_{1}}\right)+\frac{v_{\mid \Gamma_{1}}}{2}-S_{\Gamma_{1}}\left(\partial_{\nu} v_{\mid \Gamma_{1}}\right), \\
\partial_{\nu} v_{\mid \Gamma_{1}} & =T_{\Gamma_{1}}\left(v_{\mid \Gamma_{1}}\right)-K_{\Gamma_{1}}^{\prime}\left(\partial_{\nu} v_{\mid \Gamma_{1}}\right)+\frac{\partial_{\nu} v_{\mid \Gamma_{1}}}{2},
\end{aligned}
$$

which proves that $\left(v, \partial_{\nu} v\right)_{\mid \Gamma_{1}} \in \operatorname{Ker}\left(P_{\Gamma_{1}}\right)$. On the other hand let $(\varphi, \psi) \in \operatorname{Ker}\left(P_{\Gamma_{1}}\right)$ and let the function $v$ defined by (16). Using again trace formulas for double and single layer potentials, we obtain

$$
\left(\begin{array}{c}
v_{\mid \Gamma_{1}}^{-} \\
\partial_{\nu} v_{\mid \Gamma_{1}}^{-}
\end{array}\right)=P_{\Gamma_{1}}\left(\begin{array}{c}
\psi \\
\varphi
\end{array}\right)=\left(\begin{array}{l}
0 \\
0
\end{array}\right) .
$$

Therefore the function $v$ replaced by 0 outside $\Omega_{1}$ is a $H^{1}$ solution to the Helmholtz equation in $\mathbf{R}^{m}$. Unique continuation principle implies that $v$ is zero inside $\Omega_{1}$, which proves that $\left(v, \partial_{\nu} v\right)_{\mid \Gamma_{2}}$ is also zero. Consequently $(\varphi, \psi) \in \operatorname{Ker}\left(T_{\Gamma_{1} \rightarrow \Gamma_{2}}\right)$.

We now characterize the range of the operators $T_{\Gamma_{2} \rightarrow \Gamma_{1}}$ and $T_{\Gamma_{1} \rightarrow \Gamma_{2}}$. This is consequence of the previous lemma using a duality argument. Let us define a duality pairing $\langle,\rangle_{i}$ between $H^{1 / 2}\left(\Gamma_{i}\right) \times$ $H^{-1 / 2}\left(\Gamma_{i}\right)$ and its dual $H^{-1 / 2}\left(\Gamma_{i}\right) \times H^{1 / 2}\left(\Gamma_{i}\right)$ such that

$$
\left\langle(\varphi, \psi),\left(\varphi^{\prime}, \psi^{\prime}\right)\right\rangle_{i}:=\int_{\Gamma_{i}} \varphi \varphi^{\prime} d s-\int_{\Gamma_{i}} \psi \psi^{\prime} d s
$$

for regular functions $(\varphi, \psi)$ and $\left(\varphi^{\prime}, \psi^{\prime}\right)$. Then for $\left(\varphi_{2}, \psi_{2}\right) \in H^{1 / 2}\left(\Gamma_{2}\right) \times H^{-1 / 2}\left(\Gamma_{2}\right)$ and $\left(\varphi_{1}, \psi_{1}\right) \in$ $H^{1 / 2}\left(\Gamma_{1}\right) \times H^{-1 / 2}\left(\Gamma_{1}\right)$,

$$
\begin{aligned}
&-\left\langle T_{\Gamma_{2} \rightarrow \Gamma_{1}}\left(\varphi_{2}, \psi_{2}\right),\right.\left.\left(\psi_{1}, \varphi_{1}\right)\right\rangle_{1} \\
&=\int_{\Gamma_{1}} \int_{\Gamma_{2}} \frac{\partial \Phi(x, y)}{\partial \nu(y)} \varphi_{2}(y) \psi_{1}(x)-\int_{\Gamma_{1}} \int_{\Gamma_{2}} \Phi(x, y) \psi_{2} \psi_{1}(x) \\
& \quad-\int_{\Gamma_{1}} \int_{\Gamma_{2}} \frac{\partial^{2} \Phi(x, y)}{\partial \nu(x) \partial \nu(y)} \varphi_{2}(y) \varphi_{1}(x)+\int_{\Gamma_{1}} \int_{\Gamma_{2}} \frac{\partial \Phi(x, y)}{\partial \nu(x)} \psi_{2}(y) \varphi_{1}(x),
\end{aligned}
$$

where the integrals should be understood as duality products. Observing the symmetry between the indexes 1 and 2 in this identity we conclude

$$
\left\langle T_{\Gamma_{2} \rightarrow \Gamma_{1}}\left(\varphi_{2}, \psi_{2}\right),\left(\psi_{1}, \varphi_{1}\right)\right\rangle_{1}=\left\langle T_{\Gamma_{1} \rightarrow \Gamma_{2}}\left(\varphi_{1}, \psi_{1}\right),\left(\psi_{2}, \varphi_{2}\right)\right\rangle_{2} .
$$

Similarly, for $(\varphi, \psi) \in H^{1 / 2}\left(\Gamma_{i}\right) \times H^{-1 / 2}\left(\Gamma_{i}\right)$ and $(\tilde{\varphi}, \tilde{\psi}) \in H^{1 / 2}\left(\Gamma_{i}\right) \times H^{-1 / 2}\left(\Gamma_{i}\right)$

$$
\begin{aligned}
\left\langle P_{\Gamma_{i}}(\varphi, \psi),(\tilde{\psi}, \tilde{\varphi})\right\rangle_{i}=\int_{\Gamma_{i}}-K_{\Gamma_{i}} \varphi \tilde{\psi}+\frac{1}{2} \varphi \tilde{\psi}+S_{\Gamma_{i}} \psi \tilde{\psi} d x & \\
& -\int_{\Gamma_{i}}-T_{\Gamma_{i}} \varphi \tilde{\varphi}+K_{\Gamma_{i}}^{\prime} \psi \tilde{\varphi}+\frac{1}{2} \psi \tilde{\varphi} d x .
\end{aligned}
$$

We deduce that

$$
\left\langle P_{\Gamma_{i}}(\varphi, \psi),(\tilde{\psi}, \tilde{\varphi})\right\rangle_{i}=\left\langle\left(P_{\Gamma_{i}}-I_{\Gamma_{i}}\right)(\tilde{\varphi}, \tilde{\psi}),(\psi, \varphi)\right\rangle_{i} \quad i=1,2 .
$$

These duality identities allows us to deduce the following corollary. 
Corollary 4. The following identities hold.

$$
\begin{aligned}
& \overline{\text { Range }\left(T_{\Gamma_{2} \rightarrow \Gamma_{1}}\right)}=\operatorname{Range}\left(P_{\Gamma_{1}}-I_{\Gamma_{1}}\right)=\operatorname{Ker}\left(P_{\Gamma_{1}}\right), \\
& \overline{\operatorname{Range}\left(T_{\Gamma_{1} \rightarrow \Gamma_{2}}\right)}=\operatorname{Range}\left(P_{\Gamma_{2}}\right)=\operatorname{Ker}\left(P_{\Gamma_{2}}-I_{\Gamma_{2}}\right) .
\end{aligned}
$$

Proof. Identity (17) shows that

$$
\overline{\operatorname{Range}\left(T_{\Gamma_{2} \rightarrow \Gamma_{1}}\right)}=\left(\operatorname{Ker}\left(T_{\Gamma_{1} \rightarrow \Gamma_{2}}\right)\right)^{\perp} \text { and } \overline{\operatorname{Range}\left(T_{\Gamma_{1} \rightarrow \Gamma_{2}}\right)}=\left(\operatorname{Ker}\left(T_{\Gamma_{2} \rightarrow \Gamma_{1}}\right)\right)^{\perp}
$$

where the orthogonality is with respect to the duality products $\langle., .\rangle_{i}$ defined. We also have using (18)

$$
\operatorname{Ker}\left(P_{\Gamma_{i}}^{\perp}\right)=\operatorname{Range}\left(P_{\Gamma_{i}}-I_{\Gamma_{i}}\right)=\operatorname{Ker}\left(P_{\Gamma_{i}}\right) .
$$

The announced identities are then be deduced as direct consequences of those in Lemma 3 .

We are now in position to state the main result. We define

$$
X\left(\Gamma_{i}\right):=H^{1 / 2}\left(\Gamma_{i}\right) \times H^{-1 / 2}\left(\Gamma_{i}\right)
$$

and the subspace of $X\left(\Gamma_{1}\right) \times X\left(\Gamma_{2}\right)$

$$
Y\left(\Gamma_{1} \times \Gamma_{2}\right):=\operatorname{Ker}\left(P_{\Gamma_{1}}\right) \times \operatorname{Ker}\left(P_{\Gamma_{2}}-I_{\Gamma_{2}}\right) .
$$

Theorem 5. The operator $A: X\left(\Gamma_{2}\right) \rightarrow Y\left(\Gamma_{1} \times \Gamma_{2}\right)$ defined by expression (11) is one to one and has a dense range. Moreover $\overline{\operatorname{Range}(A)}=\overline{\operatorname{Range}(B)}$ where $B$ is defined by (12).

Proof. We start by proving the injectivity of the operator $A$. Since $P_{\Gamma_{2}}$ is a projector then

$$
\text { for all } u \in X\left(\Gamma_{2}\right), \quad u=u_{1}+u_{2}
$$

with $u_{1} \in \operatorname{Ker}\left(P_{\Gamma_{2}}\right)$ and $u_{2} \in \operatorname{Range}\left(P_{\Gamma_{2}}\right)$. Assume that $A(u)=0$. Then

$$
T_{\Gamma_{2} \rightarrow \Gamma_{1}}(u)=0 \quad \text { and } \quad P_{\Gamma_{2}}(u)=0 .
$$

Since Range $\left(P_{\Gamma_{2}}\right)=\operatorname{Ker}\left(P_{\Gamma_{2}}-I_{\Gamma_{2}}\right)$, we get from Lemma 3 that $u_{2} \in \operatorname{Ker}\left(T_{\Gamma_{2} \rightarrow \Gamma_{1}}\right)$. Consequently $T_{\Gamma_{2} \rightarrow \Gamma_{1}}\left(u_{1}\right)=0$ and therefore $u_{1} \in \operatorname{Range}\left(P_{\Gamma_{2}}\right)$. This implies $u_{1}=0$.

On the other hand, $A(u)=0$ also implies $P_{\Gamma_{2}}(u)=0$ and therefore $P_{\Gamma_{2}}\left(u_{2}\right)=0$. This proves that $u_{2}=0$ since we already have $u_{2} \in \operatorname{Range}\left(P_{\Gamma_{2}}\right)$. The injectivity of $A$ is then proved.

For the characterization of the range of the operator $A$ we use Corollary 4 in which we have proved that $\overline{\text { Range }\left(T_{\Gamma_{2} \rightarrow \Gamma_{1}}\right)}=\operatorname{Ker}\left(P_{\Gamma_{1}}\right)$. Consequently

$$
\overline{\operatorname{Range}(A)}=\operatorname{Ker}\left(P_{\Gamma_{1}}\right) \times \operatorname{Range}\left(P_{\Gamma_{2}}\right)=\operatorname{Ker}\left(P_{\Gamma_{1}}\right) \times \operatorname{Ker}\left(P_{\Gamma_{2}}-I_{\Gamma_{2}}\right)
$$

Using Corollary 4 we also deduce that

$$
\overline{\operatorname{Range}(B)}=\operatorname{Ker}\left(P_{\Gamma_{1}}\right) \times \overline{\operatorname{Range}\left(T_{\Gamma_{1} \rightarrow \Gamma_{2}}\right)}=\operatorname{Ker}\left(P_{\Gamma_{1}}\right) \times \operatorname{Ker}\left(P_{\Gamma_{2}}-I_{\Gamma_{2}}\right)=\overline{\operatorname{Range}(A)} .
$$

A straightforward consequence of this result is that any regular regularization strategy can be applied to equation 13 in the case of noisy data. We hereafter state the result for the case of Tikhonov regularization. 
Corollary 6. Let $(f, g) \in X\left(\Gamma_{1}\right)$ and $\left(u_{\mid \Gamma_{2}}, \partial_{\nu} u_{\mid \Gamma_{2}}\right) \in X\left(\Gamma_{2}\right)$ such that

$$
A\left(\begin{array}{c}
u_{\mid \Gamma_{2}} \\
\partial_{\nu} u_{\mid \Gamma_{2}}
\end{array}\right)=B\left(\begin{array}{c}
f \\
g
\end{array}\right)
$$

and let $\left(f^{\delta}, g^{\delta}\right) \in X\left(\Gamma_{1}\right)$ such that

$$
\left\|\left(\begin{array}{c}
f^{\delta} \\
g^{\delta}
\end{array}\right)-\left(\begin{array}{c}
f \\
g
\end{array}\right)\right\|_{X\left(\Gamma_{1}\right)}<\delta
$$

Consider the Tikhonov solution $\left(u^{\delta}, \partial_{\nu} u^{\delta}\right)$ satisfying

$$
\left(\alpha(\delta) I_{\Gamma_{2}}+A^{*} A\right)\left(\begin{array}{c}
u^{\delta} \\
\partial_{\nu} u^{\delta}
\end{array}\right)=A^{*} B\left(\begin{array}{c}
f^{\delta} \\
g^{\delta}
\end{array}\right)
$$

where $\alpha(\delta)$ is determined (for sufficiently small $\delta$ ) using the Morozov discrepancy principle, i.e $\alpha(\delta)$ is the unique solution of

$$
\left\|A\left(\begin{array}{c}
u^{\delta} \\
\partial_{\nu} u^{\delta}
\end{array}\right)-B\left(\begin{array}{c}
f^{\delta} \\
g^{\delta}
\end{array}\right)\right\|_{Y\left(\Gamma_{1} \times \Gamma_{2}\right)}=\delta\|B\|_{X\left(\Gamma_{1}\right) \rightarrow Y\left(\Gamma_{1} \times \Gamma_{2}\right)} .
$$

Then

$$
\left\|\left(\begin{array}{c}
u^{\delta} \\
\partial_{\nu} u^{\delta}
\end{array}\right)-\left(\begin{array}{c}
u \\
\partial_{\nu} u
\end{array}\right)\right\|_{X\left(\Gamma_{2}\right)} \longrightarrow 0 \quad \text { as } \quad \delta \longrightarrow 0 .
$$

Proof. The proof of this Corollary can be deduced directly from Theorem 5 using the Tikhonov regularization theory and the Morozov discrepancy principle (See for instance [13, Theorem 4.16])

In the previous corollary, one assumes that $f^{\delta} \in H^{1 / 2}\left(\Gamma_{1}\right)$. A more reasonable assumption would be that the noisy data is only in $L^{2}\left(\Gamma_{1}\right)$. In that case a regularization of the data is needed. We hereafter explain a possible method based again on Tikhonov regularization (any other standard method can be indeed used). Let $\left\{e_{n} \in L^{2}\left(\Gamma_{1}\right), n=1,2 \ldots\right\}$ denote an orthonormal basis of $L^{2}\left(\Gamma_{1}\right)$ formed by the eigenvectors of the Laplace-Beltrami operator on $\Gamma_{1}$ and denote by $\sigma_{n}$ the eigenvalue associated with $e_{n}$. For $f \in H^{s}\left(\Gamma_{1}\right)$ we recall that an equivalent definition of the $H^{s}\left(\Gamma_{1}\right)$ norm is

$$
\|f\|_{H^{s}\left(\Gamma_{1}\right)}^{2}:=\sum_{j=1}^{\infty}\left(1+\sigma_{n}^{1 / 2}\right)^{2 s}\left|\left(f, e_{n}\right)_{L^{2}\left(\Gamma_{1}\right)}\right|^{2} .
$$

Set $\lambda_{n}:=1 / \sqrt{1+\sigma_{n}^{1 / 2}}$ and set for $h \in L^{2}\left(\Gamma_{1}\right)$

$$
R_{\beta} h:=\sum_{n=1}^{\infty} \frac{\lambda_{n}}{\sqrt{\beta+\lambda_{n}^{2}}}\left(h, e_{n}\right)_{L^{2}\left(\Gamma_{1}\right)} e_{n} .
$$

The operator $R_{\beta}$ is nothing but the Tikhonov regularized inverse of the compact injection $H^{1 / 2}\left(\Gamma_{1}\right) \rightarrow$ $L^{2}\left(\Gamma_{1}\right)$. In particular, one easily verifies that for $f \in H^{1 / 2}\left(\Gamma_{1}\right)$

$$
\left\|R_{\beta} f-f\right\|_{H^{1 / 2}\left(\Gamma_{1}\right)} \rightarrow 0 \text { as } \beta \rightarrow 0
$$

and if $f \in H^{1}\left(\Gamma_{1}\right)$ then

$$
\left\|R_{\beta} f-f\right\|_{H^{1 / 2}\left(\Gamma_{1}\right)} \leq \sqrt{\beta}\|f\|_{H^{1}\left(\Gamma_{1}\right)} .
$$

Morover, for $h \in L^{2}\left(\Gamma_{1}\right)$

$$
\left\|R_{\beta} h\right\|_{H^{1 / 2}\left(\Gamma_{1}\right)} \leq \frac{1}{\sqrt{\beta}}\|h\|_{L^{2}\left(\Gamma_{1}\right)} .
$$


It is then obvious that if $\beta(\delta)$ is such that $\beta(\delta) \rightarrow 0$ and $\delta^{2} / \beta(\delta) \rightarrow 0$ as $\delta \rightarrow 0$, then for $f^{\delta} \in L^{2}\left(\Gamma_{1}\right)$ such that

$$
\left\|f^{\delta}-f\right\|_{L^{2}\left(\Gamma_{1}\right)} \leq \delta
$$

one has $R_{\beta} f^{\delta} \in H^{1 / 2}\left(\Gamma_{1}\right)$ and

$$
\left\|R_{\beta} f^{\delta}-f\right\|_{H^{1 / 2}\left(\Gamma_{1}\right)} \leq \delta / \sqrt{\beta(\delta)}+\left\|R_{\beta(\delta)} f-f\right\|_{H^{1 / 2}\left(\Gamma_{1}\right)} \rightarrow 0 \text { as } \delta \rightarrow 0 .
$$

If $f \in H^{1}\left(\Gamma_{1}\right)$ and $\beta(\delta)=\delta$ then one has the more explicit estimate

$$
\left\|R_{\delta} f^{\delta}-f\right\|_{H^{1 / 2}\left(\Gamma_{1}\right)} \leq \sqrt{\delta}\left(1+\|f\|_{H^{1}\left(\Gamma_{1}\right)}\right) .
$$

One then can state the straightforward following convergence result for $L^{2}$ noisy data.

Corollary 7. Let $(f, g) \in X\left(\Gamma_{1}\right)$ and $\left(u_{\mid \Gamma_{2}}, \partial_{\nu} u_{\mid \Gamma_{2}}\right) \in X\left(\Gamma_{2}\right)$ such that (19) is satisfied and further assume that $f \in H^{1}\left(\Gamma_{1}\right)$. Let $\left(f^{\delta}, g^{\delta}\right) \in X\left(\Gamma_{1}\right)$ such that

$$
\left\|\left(\begin{array}{c}
f^{\delta} \\
g^{\delta}
\end{array}\right)-\left(\begin{array}{c}
f \\
g
\end{array}\right)\right\|_{L^{2}\left(\Gamma_{1}\right) \times L^{2}\left(\Gamma_{1}\right)}<\delta
$$

and consider the Tikhonov solution $\left(u^{\delta}, \partial_{\nu} u^{\delta}\right)$ of $(20)$ where $f^{\delta}$ is replaced with $R_{\delta} f^{\delta}$ and $\alpha(\delta)$ is determined (for sufficiently small $\delta$ ) using the Morozov discrepancy principle, i.e $\alpha(\delta)$ is the unique solution of $(21)$ with $\delta$ in the right hand side replaced by $\sqrt{\delta}\left(1+\|f\|_{H^{1}\left(\Gamma_{1}\right)}\right)$ and again $f^{\delta}$ is replaced with $R_{\delta} f^{\delta}$. Then

$$
\left\|\left(\begin{array}{c}
u^{\delta} \\
\partial_{\nu} u^{\delta}
\end{array}\right)-\left(\begin{array}{c}
u \\
\partial_{\nu} u
\end{array}\right)\right\|_{X\left(\Gamma_{2}\right)} \longrightarrow 0 \quad \text { as } \quad \delta \longrightarrow 0 .
$$

\section{Numerical validation}

The numerical experiments are conducted in a $2 D$ setting of the problem. We recall that our algorithm is based on solving equation (20). We assume that $\Gamma_{i}$ is parametrized as

$$
\Gamma_{i}=\left\{\left(x_{i}(\theta), y_{i}(\theta)\right) ; \theta \in[0,2 \pi]\right\}
$$

with regular functions $x_{i}$ and $y_{i}, i=1,2$. Then matrix approximations $A_{M} \in \mathbf{C}^{4 M \times 2 M}$ and $B_{M} \in \mathbf{C}^{4 M \times 2 M}$ of the operators $A$ and $B$ are constructed using $M$ equally distant points $\theta_{\ell}$, $\ell=1, \ldots M$ with a step $=2 \pi / M$. We employ a collocation method and a Nyström method (with weighted trigonometric interpolation) (see [13]) to approximate the singular parts of the potentials while a trapezoidal rule is used to approximate the nonsingular component of these operators.

For a given (noisy) discrete data $F_{1} \in \mathbf{C}^{2 M}$ on the exterior boundary $\Gamma_{1}$ (that reflect pointwise values of $(f, g)$ at $\left.\left(x_{1}\left(\theta_{\ell}\right), y_{1}\left(\theta_{\ell}\right)\right)\right)$, we construct an approximation of the unknown Cauchy data $\left.F_{2} \simeq\left(u, \partial_{\nu} u\right)\right|_{\Gamma_{2}}$ using the following expression

$$
F_{2}(\ell)=\sum_{k=1}^{2 M} \frac{\sigma_{k}}{\alpha+\sigma_{k}^{2}}\left(B_{M} F_{1}, t_{k}\right) p_{k}(\ell), \quad 1 \leq \ell \leq 2 M
$$

where $\left\{\sigma_{k}, p_{k}, t_{k}\right\}_{1 \leq k \leq 2 M}$ are the $2 \mathrm{M}$ first components of the singular value decomposition of $A_{M}$ that satisfy $A_{M} p_{k}=\sigma_{k} t_{k}$, with $\sigma_{k}>0, p_{k} \in \mathbf{C}^{2 M}$ and $t_{k} \in \mathbf{C}^{4 M}$ and $\alpha$ is the regularization parameter chosen by the Morozov discrepancy principle by solving (21). In the numerical examples below we simply used (obvious) piecewise linear interpolation of data corrupted with random noise (without regularization) which already give a $H^{1}\left(\Gamma_{1}\right)$ function.

In order to construct a synthetic data that does not coincide with Helmholtz (or Laplace) solutions in all of $\Omega_{1}$ we consider an inclusion $\Omega_{0}$ (with boundary $\Gamma_{0}$ ) strictly included in $\Omega_{2}$ and construct the data from solutions to the Helmholtz (or Laplace) equation in $\Omega_{1} \backslash \Omega_{0}$ with Neumann boundary 
conditions on $\Gamma_{0}$ and a prescribed Dirichlet data on $\Gamma_{1}$. A numerical solution is obtained using a boundary integral equation formulation of the problem approximated with a Nyström method (and weighted trigonometric interpolation quadratures on an equidistant mesh, as explained in $[13$, Chapter 3]).

In the first test, we consider as prescribed Dirichlet data on $\Gamma_{1}, f(x)=\Phi\left(x, x_{0}\right)$ where $x_{0}$ is a point source fixed in the exterior of $\Omega_{1}$. The parametrization of $\Gamma_{1}, \Gamma_{2}$ and $\Gamma_{0}$ are given by

$$
\begin{aligned}
& \Gamma_{1}=\{(1.2 \cos (\theta), \quad 1.5 \sin (\theta)+0.05 \sin (3 \theta))\} \\
& \Gamma_{2}=\{(0.8 \cos (\theta), \quad \sin (\theta)+0.1 \sin (3 \theta))\} \\
& \Gamma_{0}=\{(0.5 \cos (\theta)+0.1 \cos (2 \theta), \quad 0.5 \sin (\theta))\}
\end{aligned}
$$

The point source is at $(5,5)$.

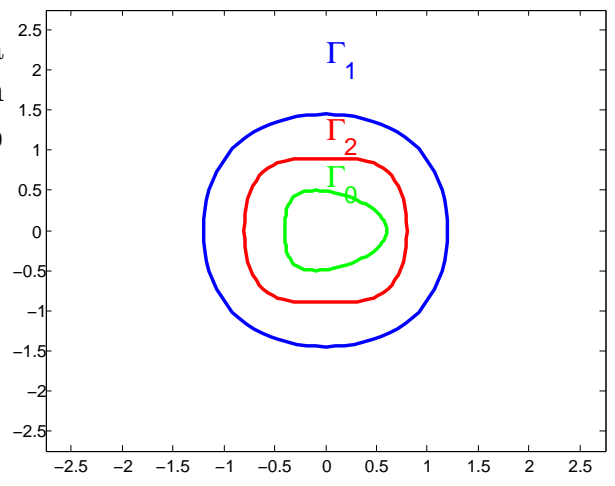

Figure 2: Sketch of the geometry for the first example.

The series of Figures $3-4-5$ show the obtained reconstructions for different values of the wave number $k=0,2,4$. We observe that a good accuracy is achieved for noise-free and noisy data. The accuracy-dependence on the frequency (corresponding with a not too small wavelength) seems to be robust.
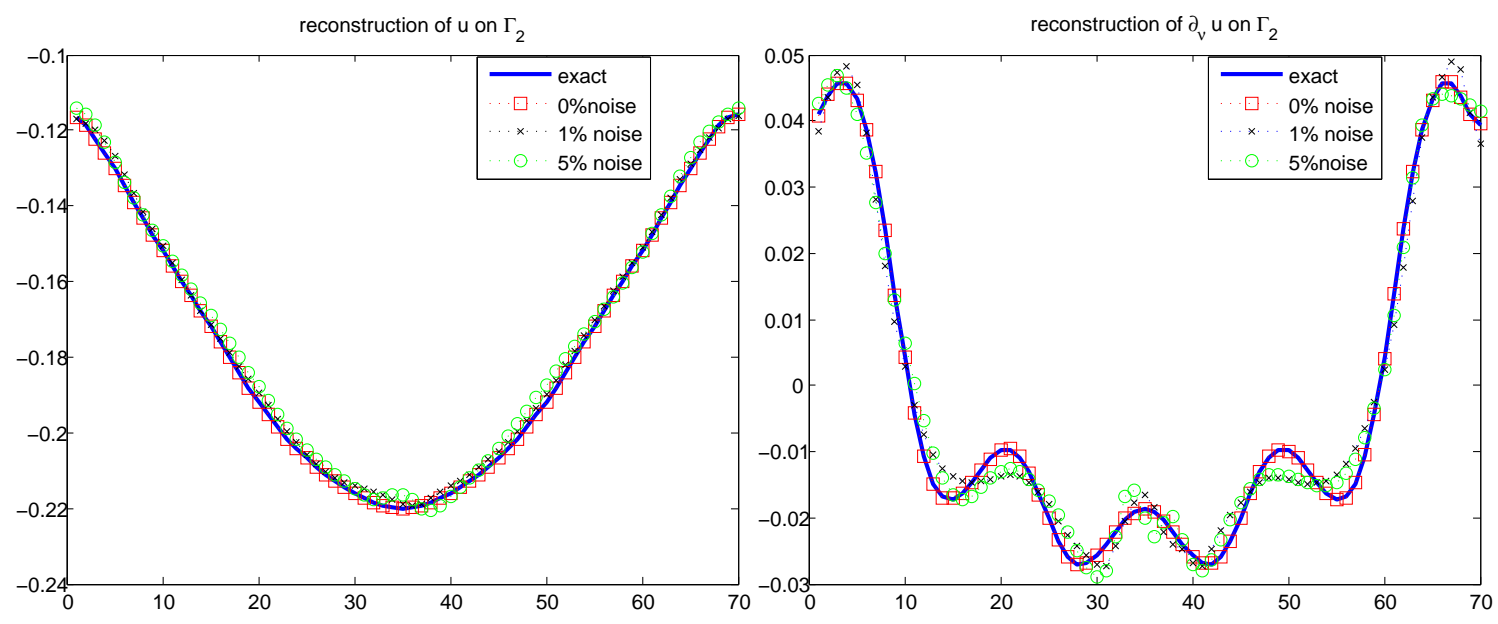

Figure 3: Reconstruction of $u$ (left) and $\partial_{\nu} u$ (right) for the test problem described in Figure 2 and for a wave number $k=0$. 

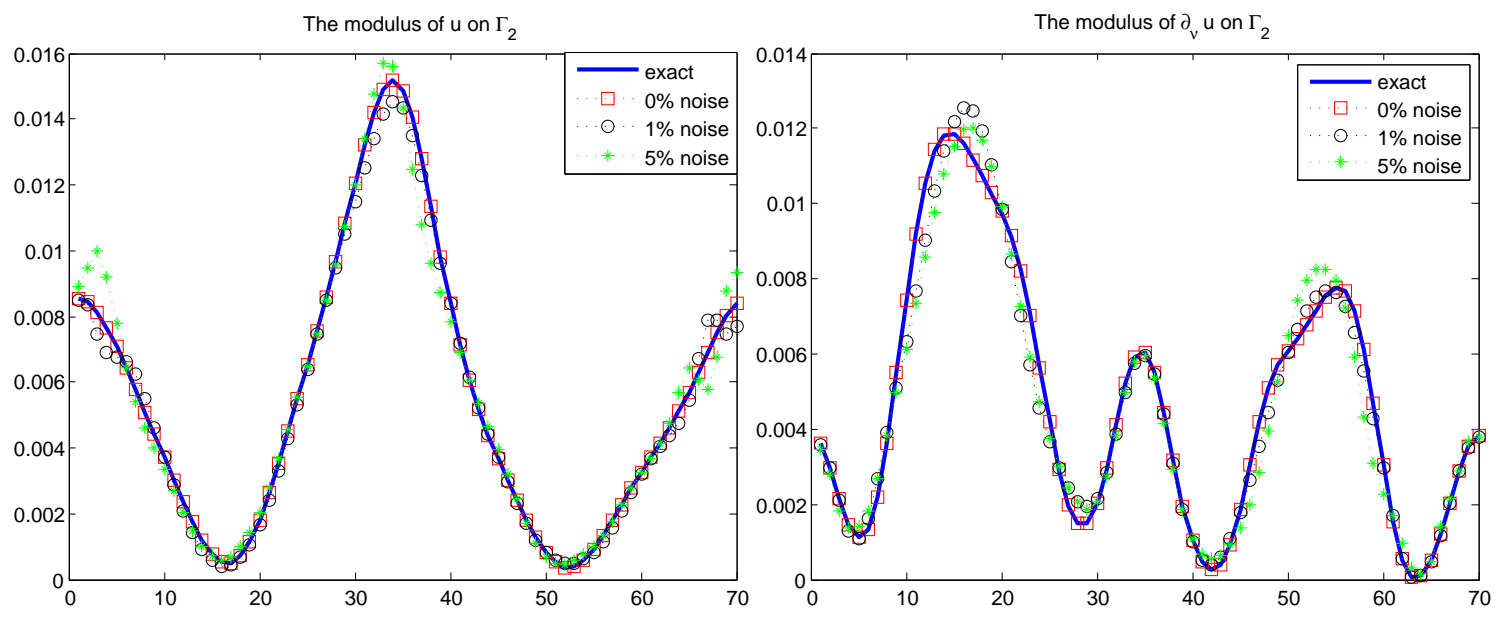

Figure 4: Reconstruction of the modulus of $u$ (left) and the modulus of $\partial_{\nu} u$ (right) for the test problem described in Figure 2 and a wave number $k=2$.
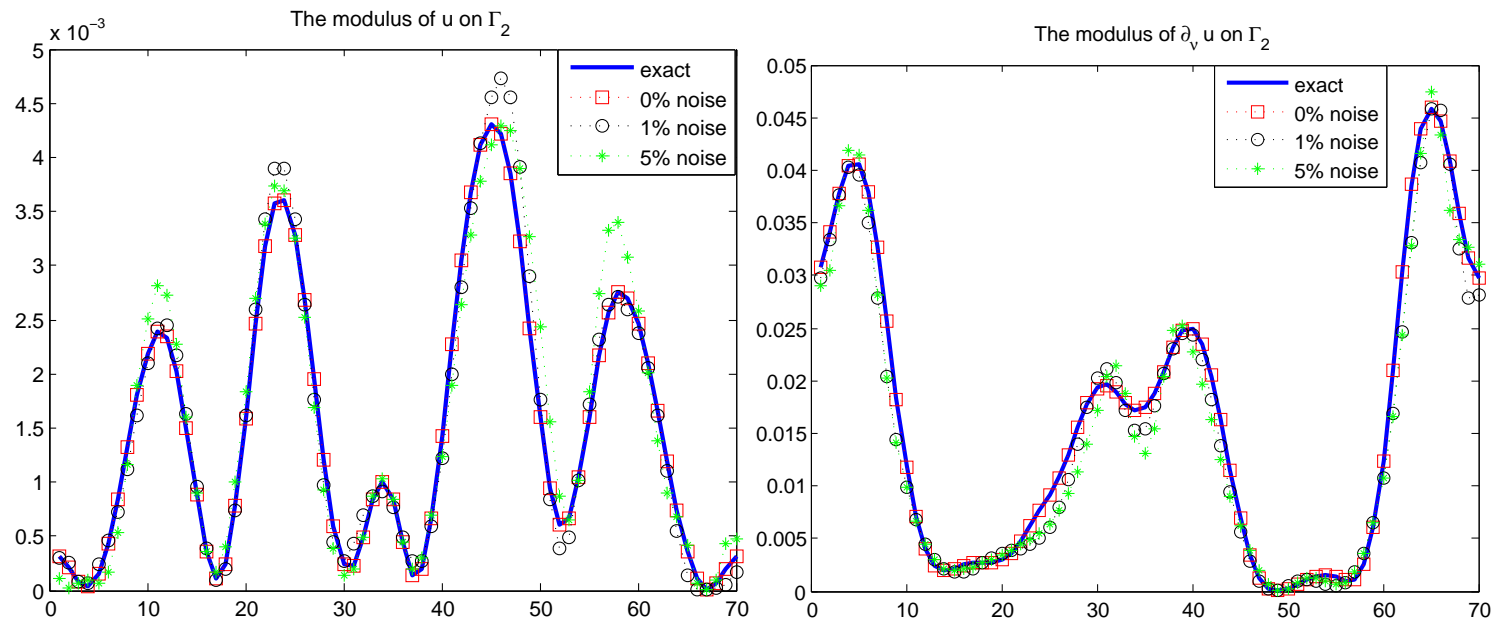

Figure 5: Reconstruction of the modulus of $u$ (left) and $\partial_{\nu} u$ (right) for the test problem described in Figure 2 and a wave number $k=4$.

In the second test (see Figures 7-8) we consider the same prescribed Dirichlet data on $\Gamma_{1}$ as before, i.e. $f(x)=1$. $\Phi\left(x, x_{0}\right)$ but change the source location into $x_{0}=(6,0)$ and change the parametrization of $\Gamma_{1}, \Gamma_{2}$ and $\Gamma_{0}$ into

$\Gamma_{1}=\{(1.5 \cos (\theta)+0.2 \cos (2 \theta), \quad 2 \sin (\theta)+0.2 \sin (2 \theta))\}$

$\left.\left.\Gamma_{2}=\{(0.8 \cos (\theta)), \quad 0.4 \sin (\theta))+0.5 \sin (2 \theta)\right)\right)$

$\left.\left.\Gamma_{0}=\{(0.4 \cos (\theta))+0.1 \cos (2 \theta)), \quad 0.6 \sin (\theta)\right)\right)$

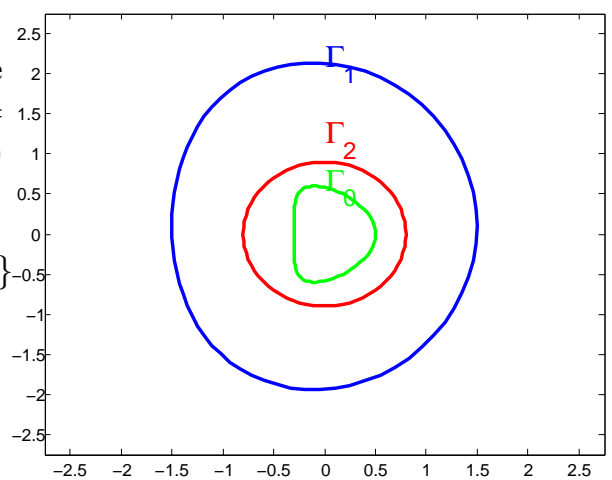

Figure 6: Sketch of the geometry for the second example. 

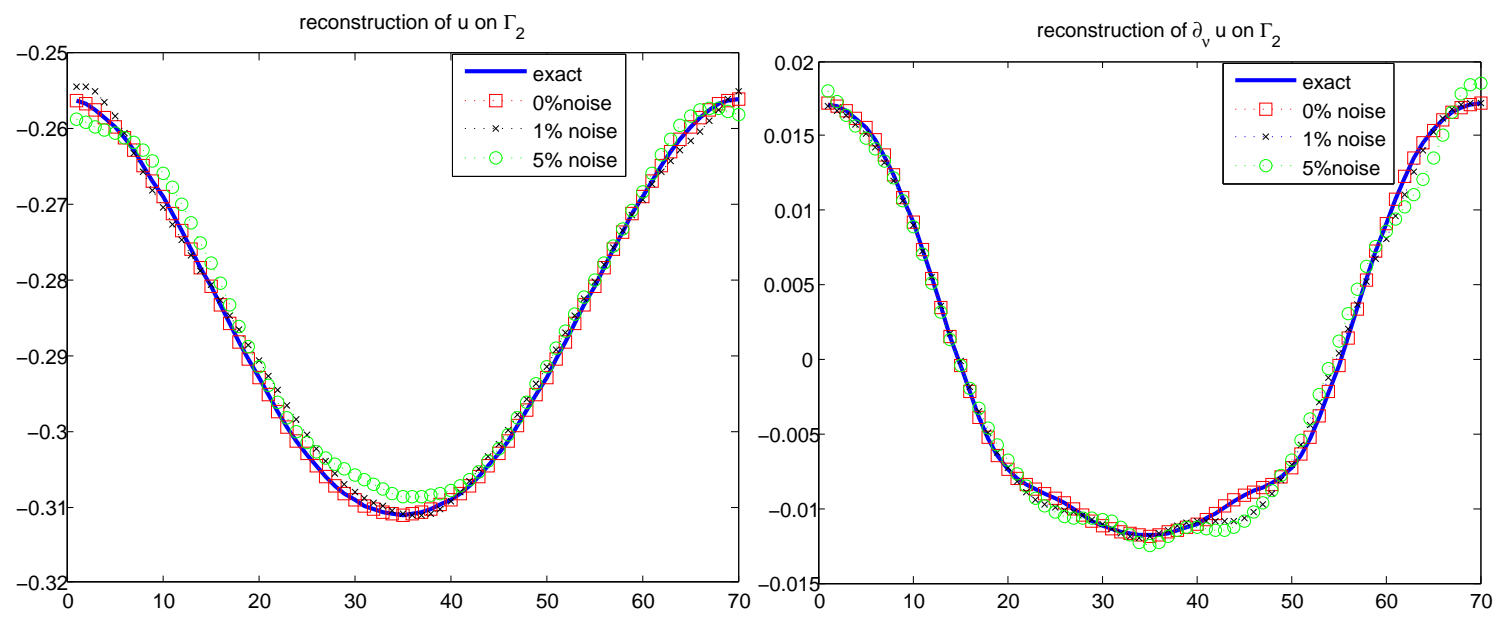

Figure 7: Reconstruction of $u$ (left) and $\partial_{\nu} u$ (right) for the test problem described in Figure 6 and a wave number $k=0$
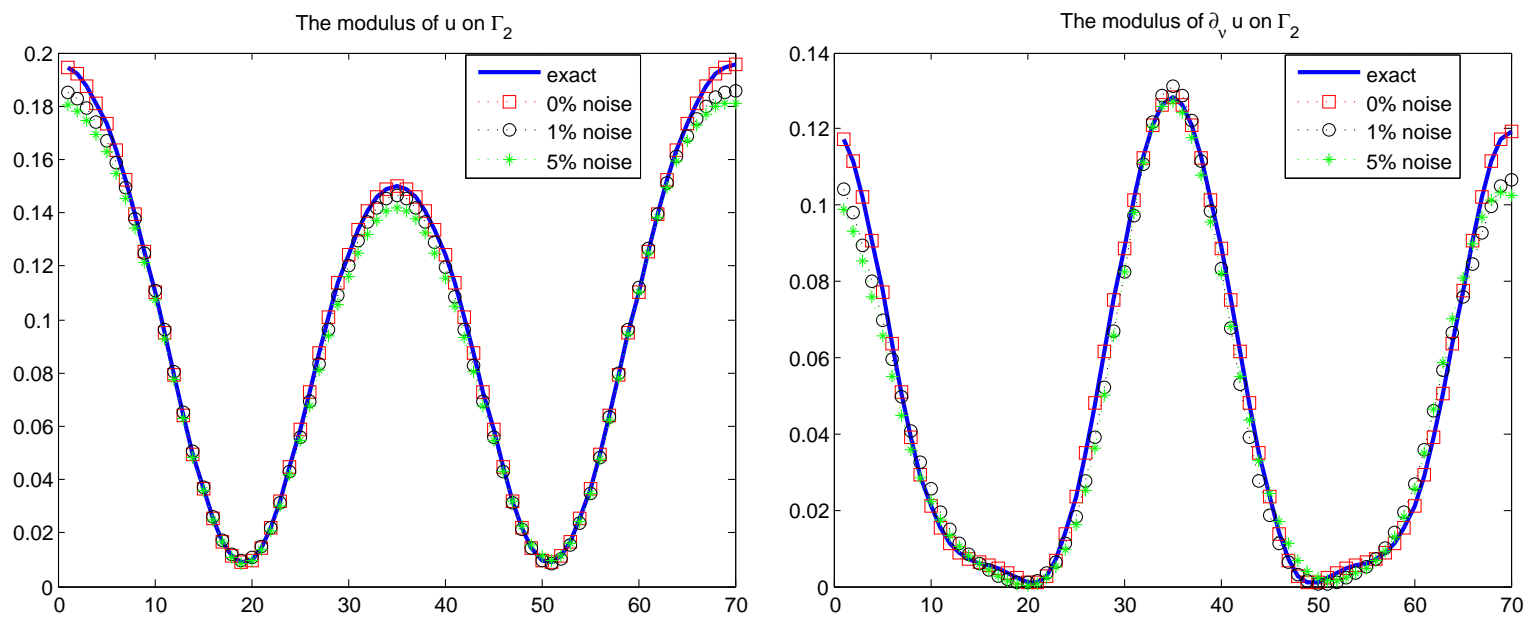

Figure 8: Reconstruction of the norm of the trace of $u$ (at left) and the norm of the trace of $\partial_{\nu} u$ (at right) for the test problem described in Figure 6 on $\Gamma_{2}$ for $k=2$

In the third test (see Figures 10-11) we consider the same setting of the second test except that the exterior boundary $\Gamma_{2}$ is slightly moved far from $\Gamma_{1}$.

The parametrization of $\Gamma_{1}, \Gamma_{2}$ and $\Gamma_{0}$ are given by

$\Gamma_{1}=\{(2 \cos (\theta)+0.2 \cos (2 \theta), \quad 2.5 \sin (\theta)+0.2 \sin (2 \theta))\}$

$\Gamma_{2}=\{(0.8 \cos (\theta), \quad 0.4 \sin (\theta)+0.5 \sin (2 \theta)\}$

$\Gamma_{0}=\{(0.4 \cos (\theta)+0.1 \cos (2 \theta), \quad 0.6 \sin (\theta))\}$

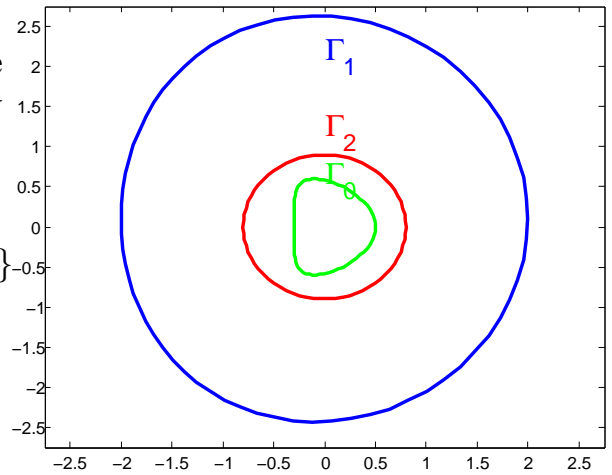

Figure 9: Sketch of the geometry for the third example. 

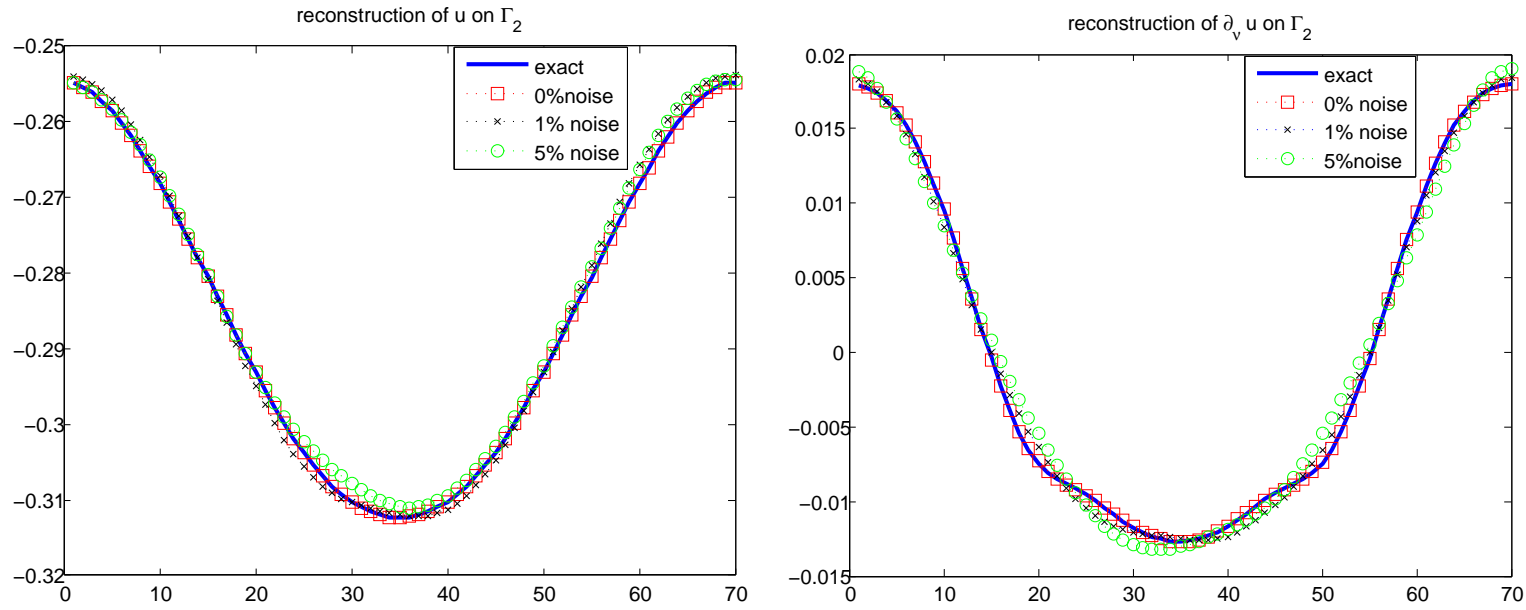

Figure 10: Reconstruction of $u$ (left) and $\partial_{\nu} u$ (right) for the test problem described in Figure 9 and a wave number $k=0$
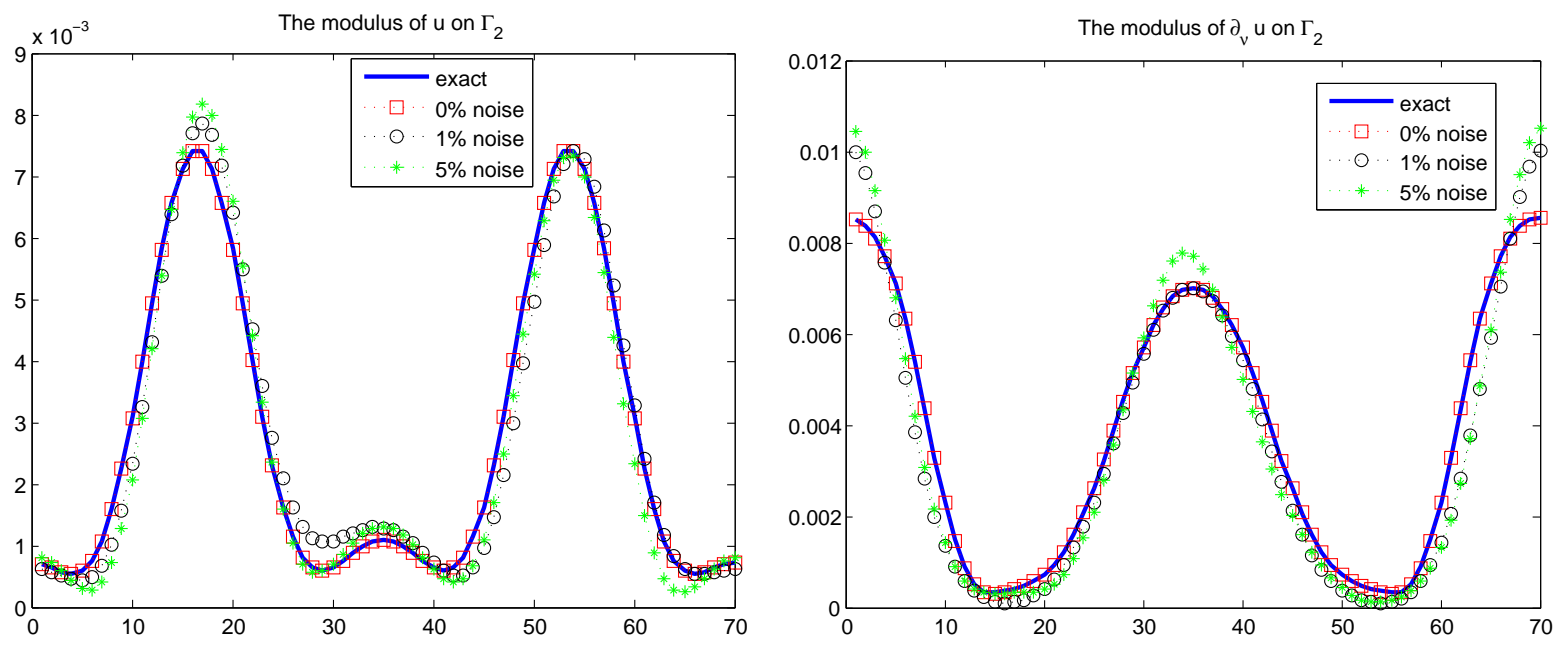

Figure 11: Reconstruction of the modulus of $u$ (left) and $\partial_{\nu} u$ (right) for the test problem described in Figure 9 and a wave number $k=2$ 
The series of Figures 7-8 and Figures 10-11 show that we have a good reconstruction achieved for different levels of noise and for different shapes of the domains. These figures show also the stability of the proposed algorithm since even by moving the exterior boundary $\Gamma_{1}$ far from $\Gamma_{2}$ the reconstruction is not very sensitive to the distance between the boundary of the given data and the boundary where data is reconstructed. In the follwing Figures 12-14 we consider the same setting as in the third test but we have changed the Dirichlet boundary conditions by imposing $f(x)=e^{i k d x}$ with $d=(0,1)$. We obtain even better reconstructions for this type data.
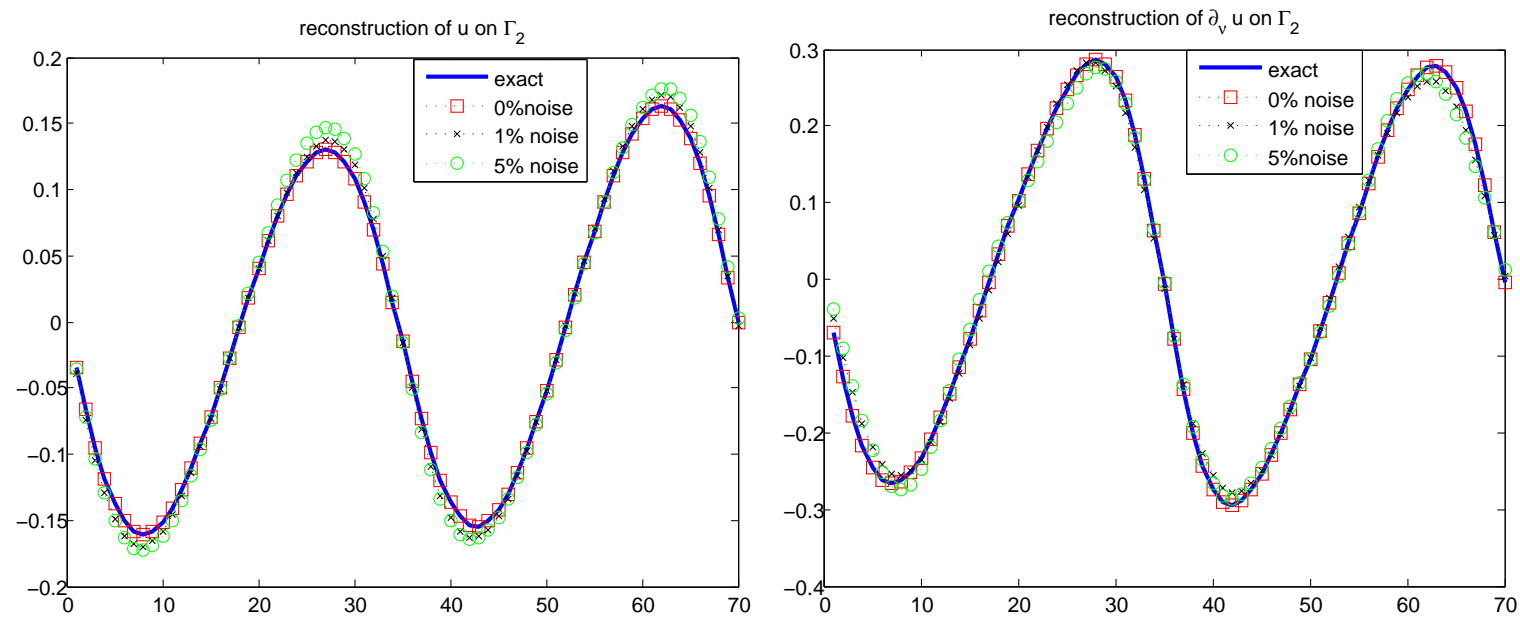

Figure 12: Reconstruction of $u$ (left) and $\partial_{\nu} u$ (right) for the test problem described in Figure 9, a wave number $k=0$ and $f(x)=e^{i k d x}$ with $d=(0,1)$.
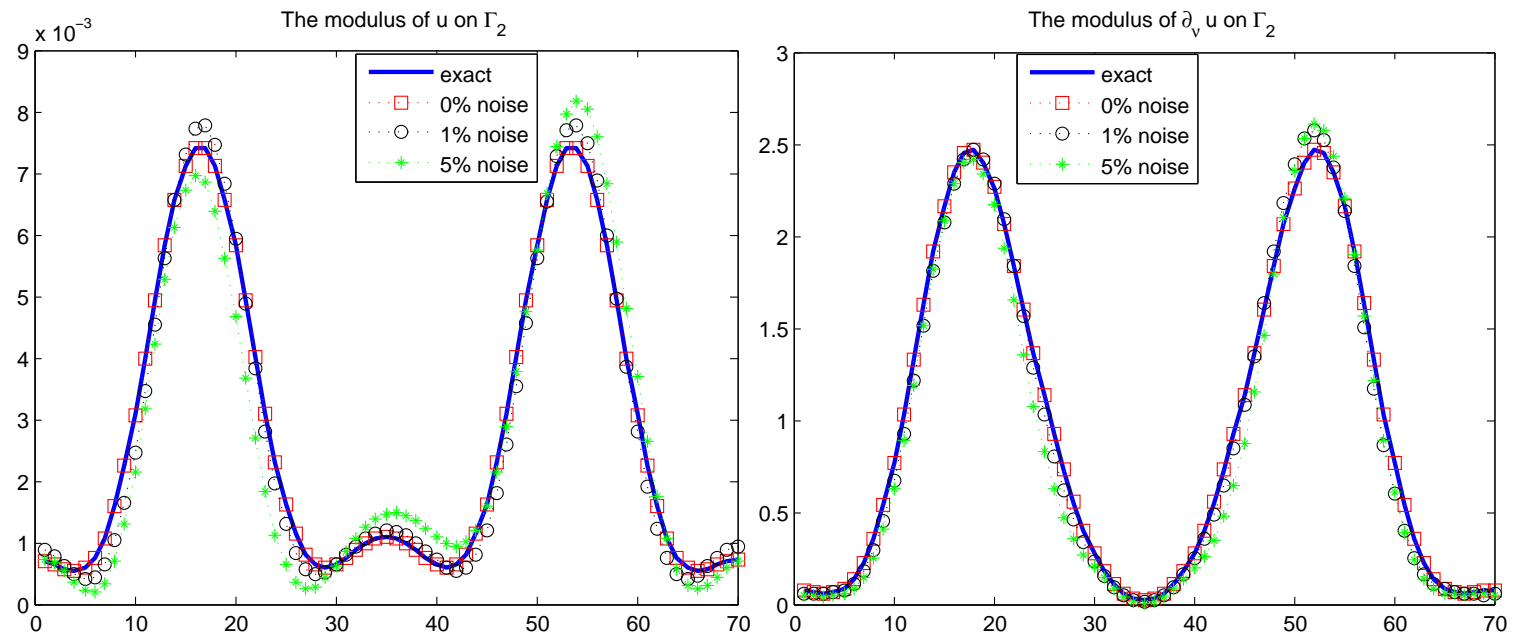

Figure 13: Reconstruction of the modulus of $u$ (left) and $\partial_{\nu} u$ (right) for the test problem described in Figure 9, a wave number $k=2$ and $f(x)=e^{i k d x}$ with $d=(0,1)$. 

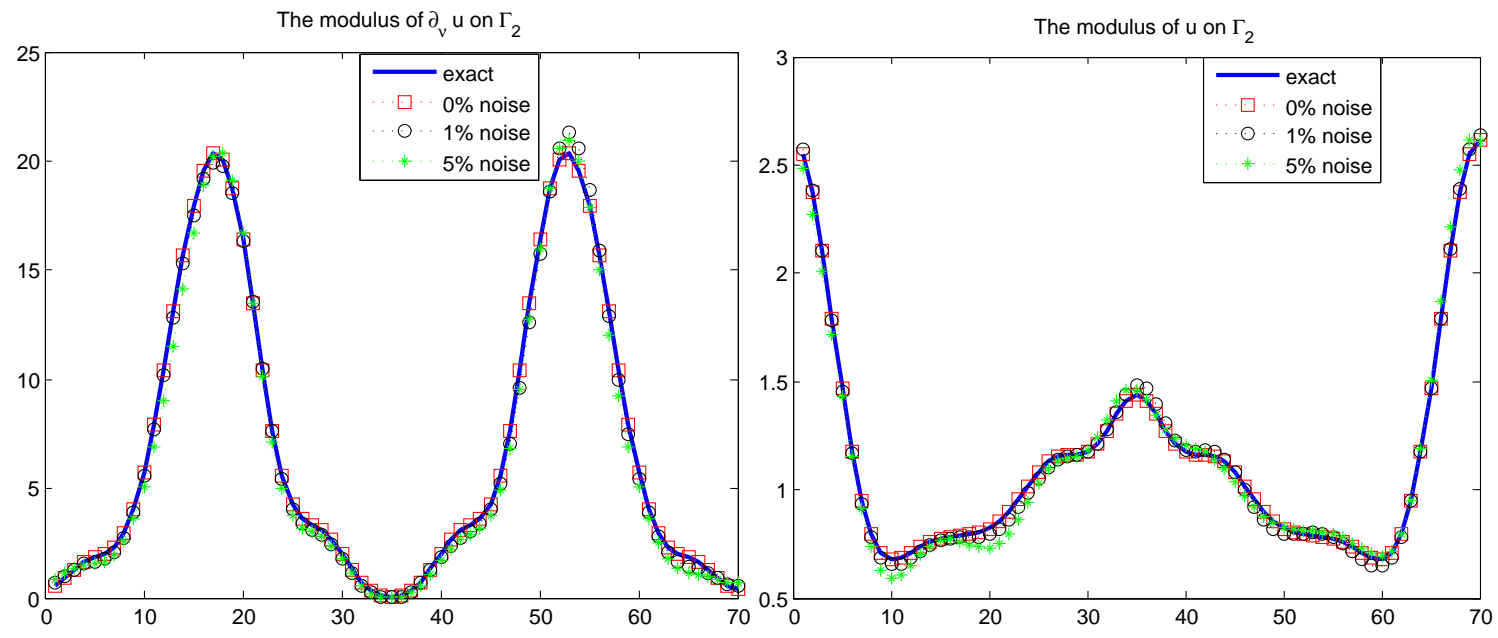

Figure 14: Reconstruction of the modulus of $u$ (left) and $\partial_{\nu} u$ (right) for the test problem described in Figure 9, a wave number $k=4$ and $f(x)=e^{i k d x}$ with $d=(0,1)$.

We now give an example of a smooth geometry where the exact solution of the Helmholtz or the Laplace equation can be analytically computed. In fact, in this test we compare the reconstructed solution with an exact solution that is known. Moroever, in the case of the Helmholz equation we compare the real and the imaginary parts of the trace of $u$ and $\partial_{\nu} u$ to show that our algorithm is also efficient to reconstruct both the modulus and the phase. Note that the conclusion also holds for previous examples where we have opted to show only the modulus for the purpose of simplifying the presentation and to provide more examples.

In the fourth test (see Figures 16-18) we will consider a smooth geometry with two boundary $\Gamma_{1}$ and $\Gamma_{2}$ defined by:

$$
\begin{aligned}
& \Gamma_{1}=\{(\cos (\theta), \quad 1.5 \sin (\theta))\} \\
& \Gamma_{2}=\{(0.5 \cos (\theta), \quad 0.75 \sin (\theta))\}
\end{aligned}
$$

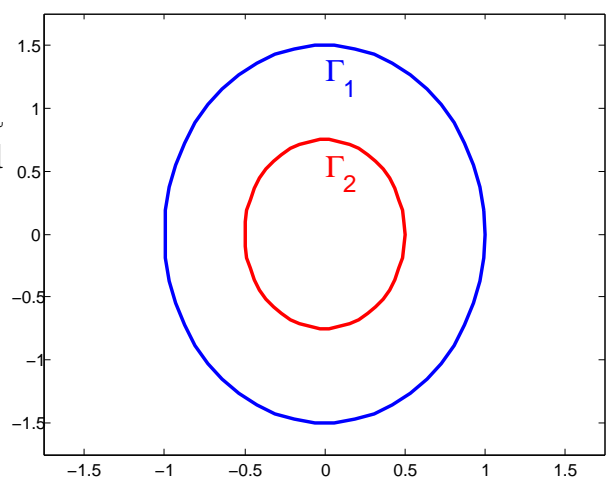

Figure 15: Sketch of the geometry for the fourth example. 

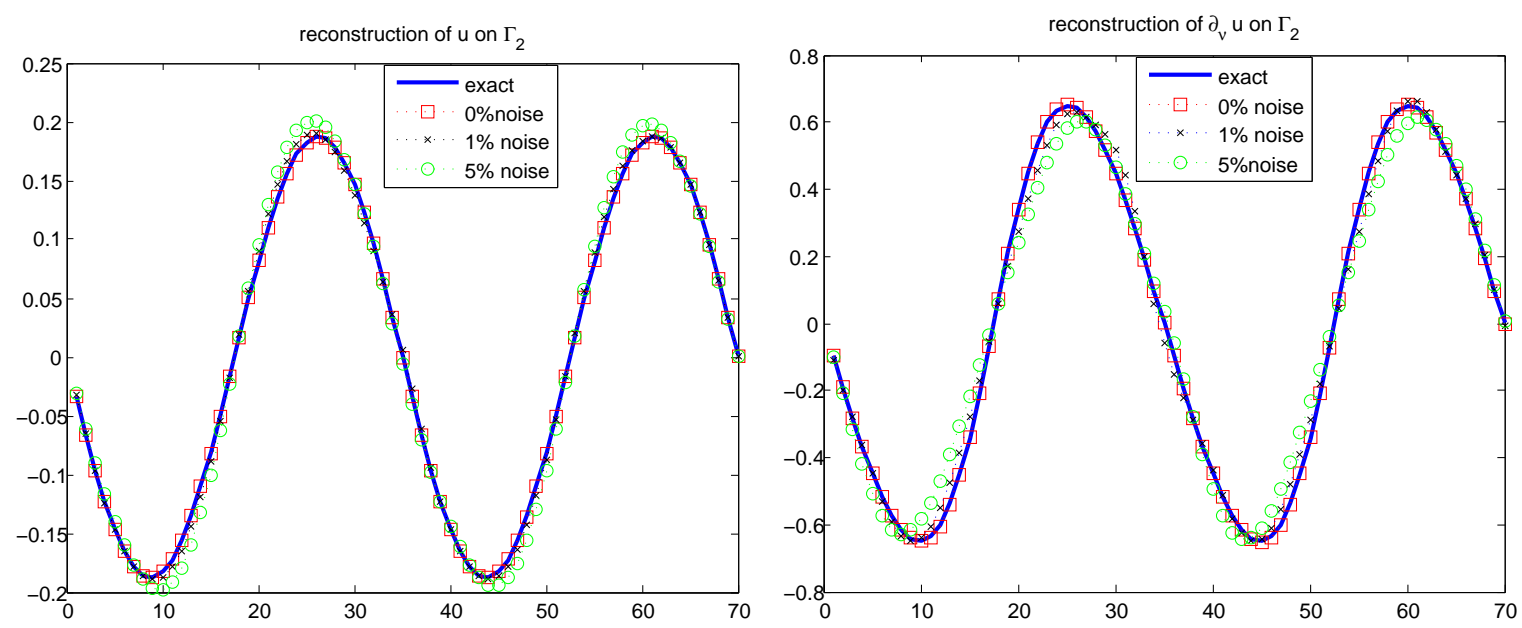

Figure 16: Reconstruction of $u$ (left) and $\partial_{\nu} u$ (right) for the test problem described in Figure 15, a wave number $k=0$ and $u(x, y)=x y$.
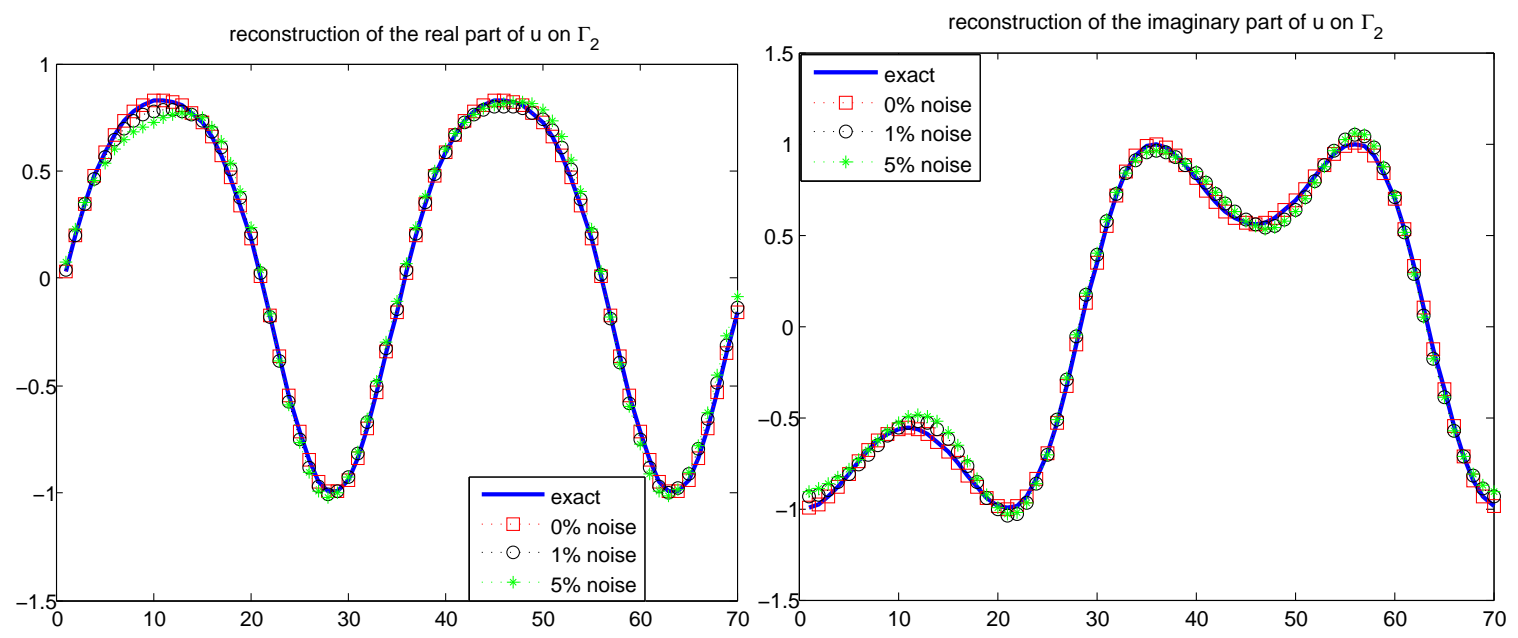

Figure 17: Reconstruction of the real part (left) and the imaginary part (right) of $u$ for the test problem described in Figure 15, a wave number $k=4$ and $f(x)=e^{i k d x}$ with $d=(\sqrt{2} / 2, \sqrt{2} / 2)$. 

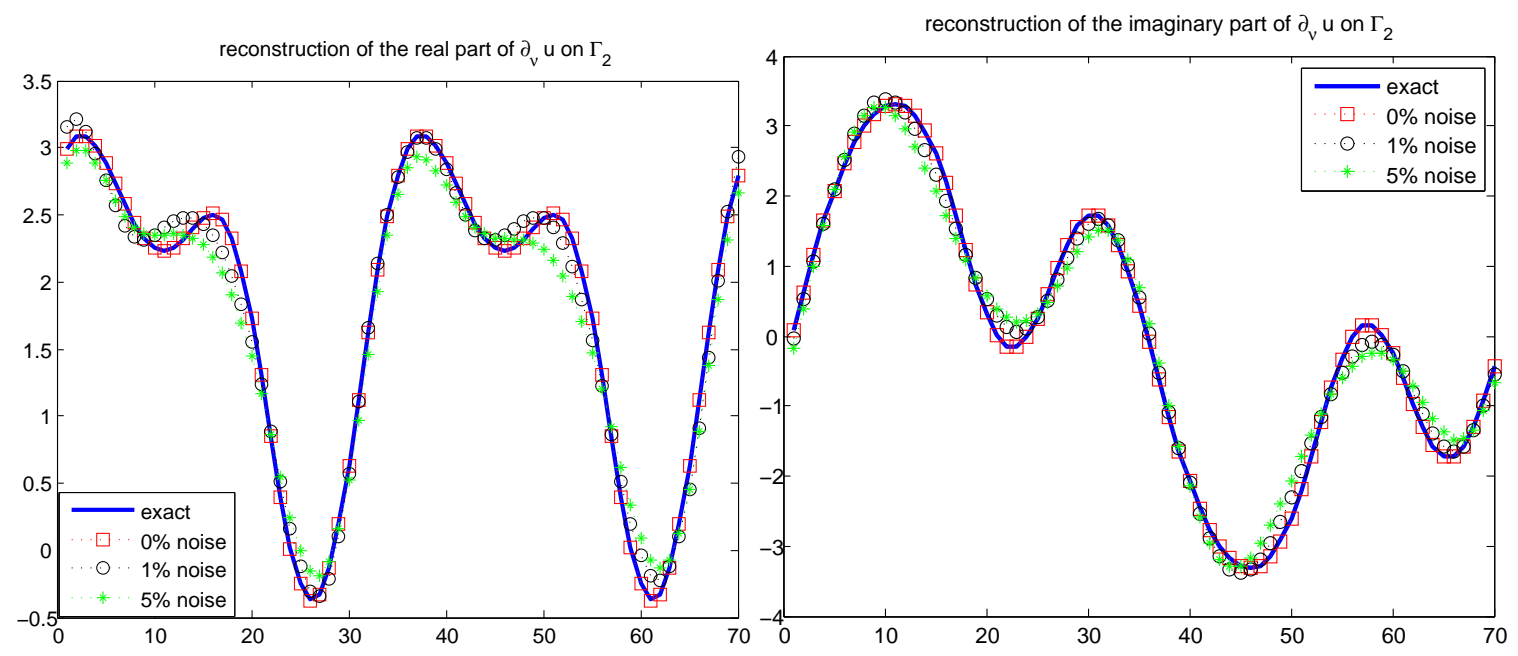

Figure 18: Reconstruction of the real part (left) and the imaginary part (right) of $\partial_{\nu} u$ for the test problem described in Figure 15, a wave number $k=4$ and $f(x)=e^{i k d x}$ with $d=(\sqrt{2} / 2, \sqrt{2} / 2)$.

We end this paper with a test where we keep the same setting of the geometry as in the last test and we suppose that we have a solution of the Helmholtz equation satisfying the Neumann boundary condition on $\Gamma_{2}$ and a Dirichlet boundary condition on $\Gamma_{1}$ with $f=\Phi\left(., x_{0}\right)$ where $x_{0}=6+5 i$.
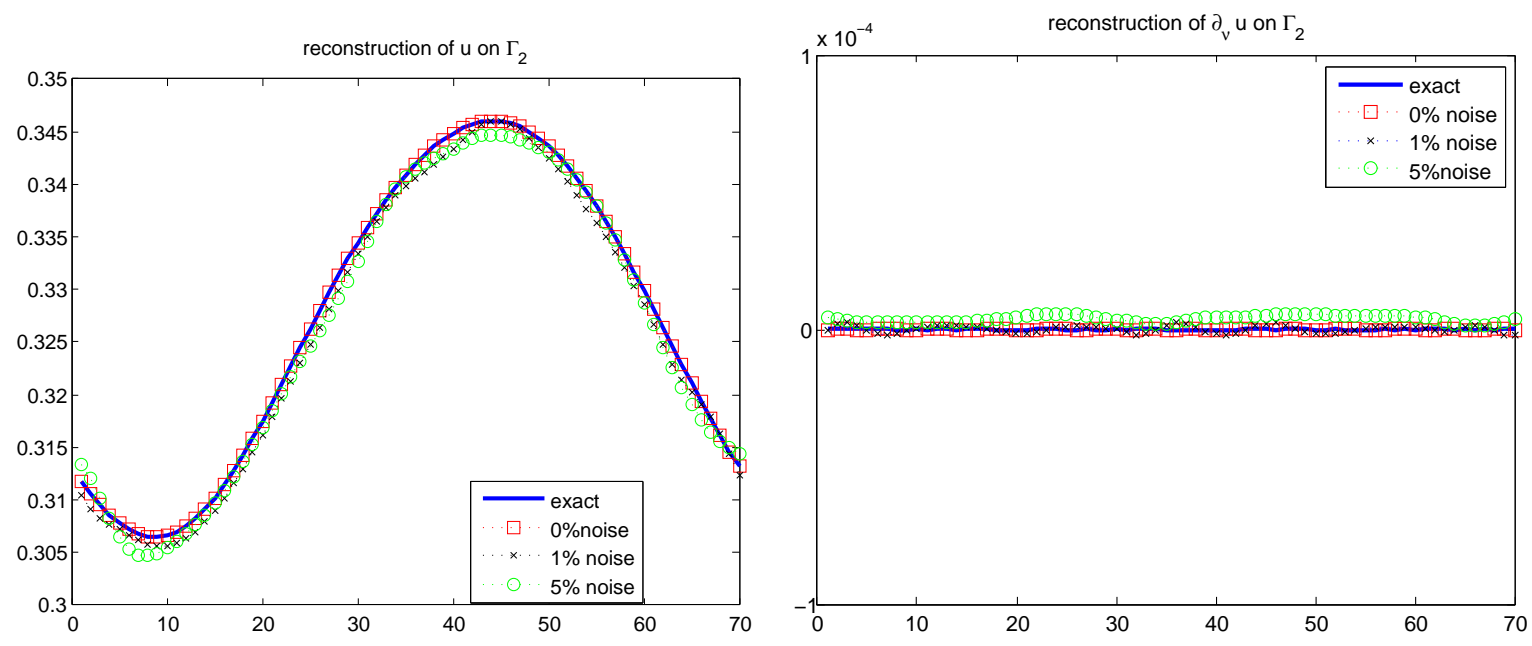

Figure 19: Reconstruction of $u$ (left) and $\partial_{\nu} u$ (right) for the test problem described in Figure 15 and a wave number $k=0$

\section{References}

[1] R. Aboulaïch, A. Ben Abda, and M. Kallel. Missing boundary data reconstruction via an approximate optimal control. Inverse Probl. Imaging, 2(4):411-426, 2008.

[2] S. Andrieux, T. N. Baranger, and A. Ben Abda. Solving Cauchy problems by minimizing an energy-like functional. Inverse Problems, 22(1):115-133, 2006. 

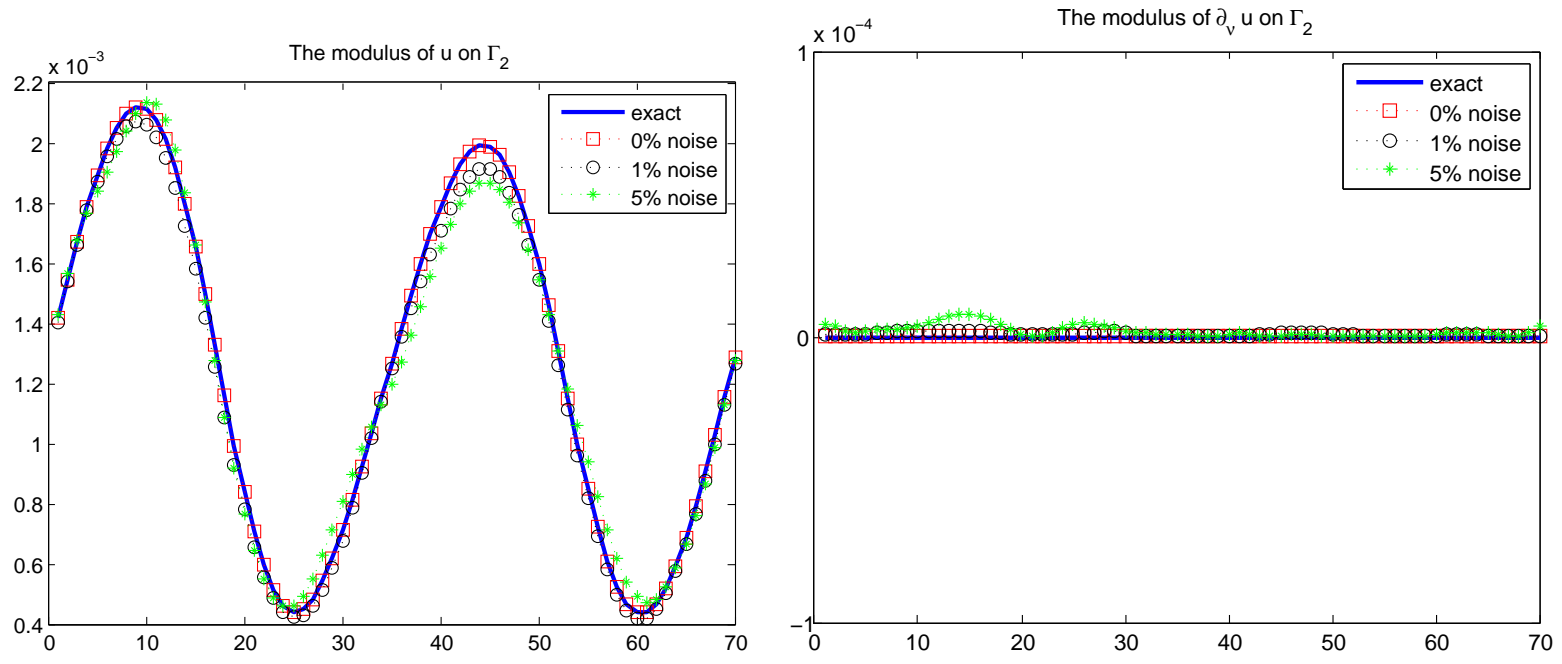

Figure 20: Reconstruction of the modulus of $u$ (left) and $\partial_{\nu} u$ (right) for the test problem described in Figure 15 and a wave number $k=2$
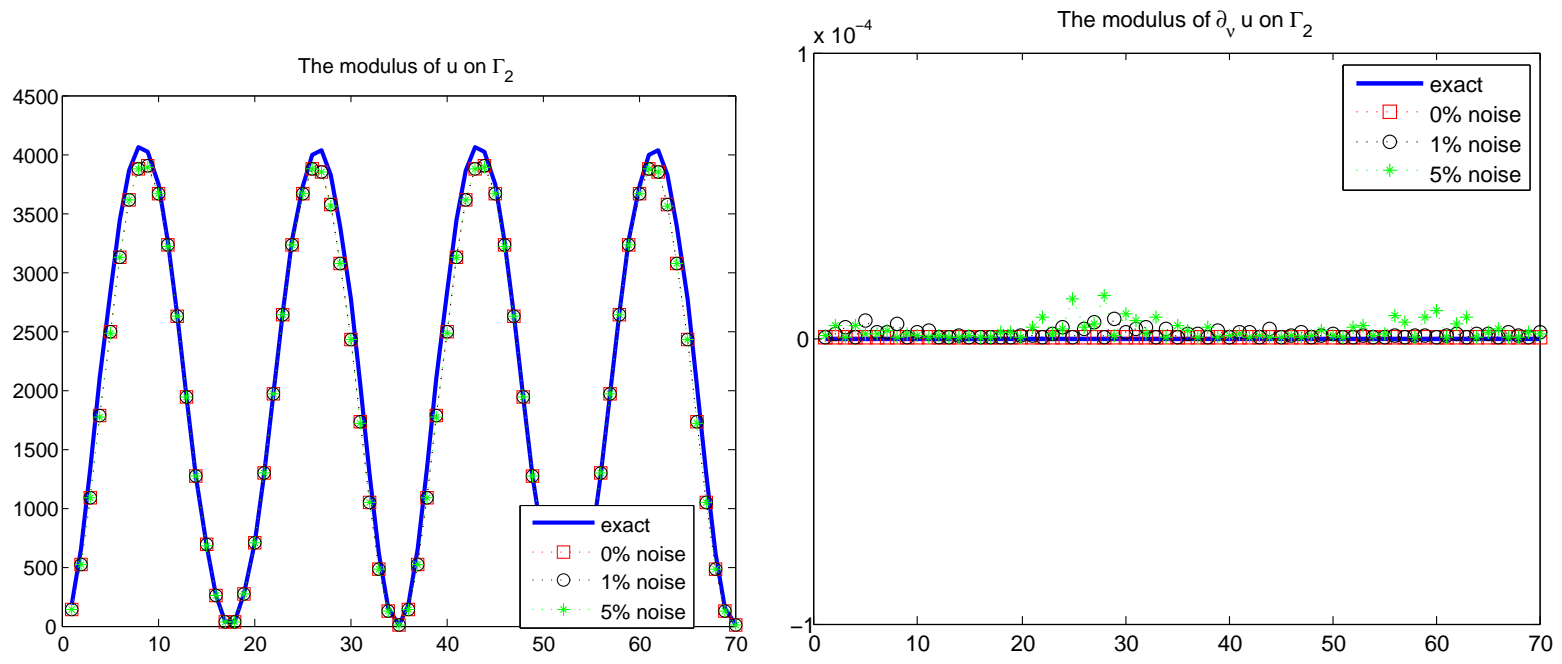

Figure 21: Reconstruction of the modulus of $u$ (left) and $\partial_{\nu} u$ (right) for the test problem described in Figure 15 and a wave number $k=4$ 
[3] A. Ben Abda, R. Ben Fatma, and D. Tromeur-Dervout. An Aitken-like acceleration method applied to missing boundary data reconstruction for the Cauchy-Helmholtz problem. C. $R$. Math. Acad. Sci. Paris, 348(1-2):93-97, 2010.

[4] F. Ben Belgacem. Why is the Cauchy problem severely ill-posed? Inverse Problems, 23(2):823-836, 2007.

[5] F. Ben Belgacem, D. Du, and F. Jelassi. Extended-domain-Lavrentiev's regularization for the Cauchy problem. Inverse Problems, 27(4):045005, 27, 2011.

[6] F. Ben Belgacem and H. El Fekih. On Cauchy's problem. I. A variational Steklov-Poincaré theory. Inverse Problems, 21(6):1915-1936, 2005.

[7] L. Bourgeois. Convergence rates for the quasi-reversibility method to solve the Cauchy problem for Laplace's equation. Inverse Problems, 22(2):413-430, 2006.

[8] L. Bourgeois. About stability and regularization of ill-posed elliptic Cauchy problems: the case of $C^{1,1}$ domains. M2AN Math. Model. Numer. Anal., 44(4):715-735, 2010.

[9] L. Bourgeois and J. Dardé. A duality-based method of quasi-reversibility to solve the Cauchy problem in the presence of noisy data. Inverse Problems, 26(9):095016, 21, 2010.

[10] F. Cakoni and R. Kress. Integral equations for inverse problems in corrosion detection from partial Cauchy data. Inverse Probl. Imaging, 1(2):229-245, 2007.

[11] H. Cao, M. V. Klibanov, and S. V. Pereverzev. A Carleman estimate and the balancing principle in the quasi-reversibility method for solving the Cauchy problem for the Laplace equation. Inverse Problems, 25(3):035005, 21, 2009.

[12] A. Cimetière, F. Delvare, M. Jaoua, and F. Pons. Solution of the Cauchy problem using iterated Tikhonov regularization. Inverse Problems, 17(3):553-570, 2001.

[13] D. Colton and R. Kress. Inverse acoustic and eletromagnetic scattering theory. Springer, New York, 2nd edition, 1998.

[14] J. Dardé, A. Hannukainen, and N. Hyvönen. An $H_{d i v}$-based mixed quasi-reversibility method for solving elliptic Cauchy problems. SIAM J. Numer. Anal., 51(4):2123-2148, 2013.

[15] A. Habbal and M. Kallel. Neumann-Dirichlet Nash strategies for the solution of elliptic Cauchy problems. SIAM J. Control Optim., 51(5):4066-4083, 2013.

[16] J. Hadamard. Lectures on Cauchy's problem in linear partial differential equations. Dover Publications, New York, 1953.

[17] H. Han and H.-J. Reinhardt. Some stability estimates for Cauchy problems for elliptic equations. J. Inverse Ill-Posed Probl., 5(5):437-454, 1997.

[18] Dinh Nho Hào and D. Lesnic. The Cauchy Problem for Laplaceś equation using the Conjugate Gradient Method. IMA J. Appl. Math, pages 199-217, 2000.

[19] Dinh Nho Hào, Phan Xuan Thanh, D. Lesnic, and B. T. Johansson. A boundary element a method for a multi-dimensional inverse heat conduction problem. Inter. J. Comput. Math., pages 1540-21554, 2012.

[20] V. Isakov and S. Kindermann. Subspaces of stability in the Cauchy problem for the Helmholtz equation. Methods Appl. Anal., 18(1):1-29, 2011.

[21] L. Jaafar Belaid, A. Ben Abda, and N. Al Malki. The Cauchy problem for the Laplace equation and application to image inpainting. ISRN Math. Anal., pages Art. ID 150979, 11, 2011. 
[22] M. V. Klibanov and F. Santosa. A computational quasi-reversibility method for Cauchy problems for Laplace's equation. SIAM J. Appl. Math., 51(6):1653-1675, 1991.

[23] V. A. Kozlov, V. G. Maz'ya, and A. V. Fomin. An iterative method for solving the Cauchy problem for elliptic equations. Zh. Vychisl. Mat. i Mat. Fiz., 31(1):64-74, 1991.

[24] R. Lattès and J.-L. Lions. Méthode de quasi-réversibilité et applications. Travaux et Recherches Mathématiques, No. 15. Dunod, Paris, 1967.

[25] L. Marin, L. Elliott, P. J. Heggs, D. B. Ingham, D. Lesnic, and X. Wen. Comparison of regularization methods for solving the Cauchy problem associated with the Helmholtz equation. Internat. J. Numer. Methods Engrg., 60(11):1933-1947, 2004.

[26] W. McLean. Strongly elliptic systems and boundary integral equations. Cambridge University Press, Cambridge, 2000.

[27] H. Reinhardt, H. Han, and Dinh Nho H. Stability and regularization of a discrete approximation to the Cauchy problem for Laplace's equation. SIAM J. Numer. Anal., 36(3):890-905, 1999 . 